\title{
Evolution of stellar collision products in open clusters ${ }^{\star}$ II. A grid of low-mass collisions
}

\author{
E. Glebbeek and O. R. Pols
}

Sterrekundig Instituut Utrecht, Postbus 80000, 3508 TA Utrecht, The Netherlands

Received 8 April 2008 / Accepted 4 June 2008

\section{ABSTRACT}

\begin{abstract}
In a companion paper we studied the detailed evolution of stellar collision products that occurred in an $N$-body simulation of the old open cluster M67 and compared our detailed models to simple prescriptions. In this paper we extend this work by studying the evolution of the collision products in open clusters as a function of mass and age of the progenitor stars.

We calculated a grid of head-on collisions covering the section of parameter space relevant for collisions in open clusters. We create detailed models of the merger remnants using an entropy-sorting algorithm and follow their subsequent evolution during the initial contraction phase, through the main sequence and up to the giant branch with our detailed stellar evolution code. We compare the location of our models in a colour-magnitude diagram to the observed blue straggler population of the old open clusters M67 and NGC 188 and find that they cover the observed blue straggler region of both clusters. For M67, collisions need to have taken place recently. Differences between the evolution tracks of the collision products and normal main sequence stars can be understood quantitatively using a simple analytic model. We present an analytic recipe that can be used in an $N$-body code to transform a precomputed evolution track for a normal star into an evolution track for a collision product.
\end{abstract}

Key words. stars: blue stragglers - stars: formation - methods: numerical - methods: $N$-body simulations Galaxy: open clusters and associations: general

\section{Introduction}

Star clusters are important laboratories for a wide range of astrophysical processes. It has become clear that the evolution of a star cluster is driven by the complex interplay between stellar dynamics and stellar and binary evolution (Portegies Zwart et al. 1999; Hurley et al. 2001). Physical collisions between stars in the dense cluster core play a pivotal role in this interaction. The products of stellar collisions between main-sequence stars potentially stand out as blue stragglers in a colour-magnitude diagram. The blue straggler population of a cluster can therefore be used to study its dynamical history. Since stellar collision products generally have a very different thermal and chemical structure than normal main-sequence stars (Lombardi et al. 1996), detailed calculations of their evolution are required (Sills et al. 1997, 2001).

In a previous paper (Glebbeek et al. 2008) we have developed an efficient procedure to import detailed models of stellar collision products into a fully implicit stellar evolution code and to evolve the remnants well beyond the main sequence. Our work is similar to that of Sills et al. (1997) but our code is faster and much more robust, allowing for the first time a systematic study of stellar merger remnants and an exploration of the parameter space. Glebbeek et al. (2008) studied the evolution of collision remnants that occurred in the $N$-body simulation of M 67 by Hurley et al. (2005) and compared these with the evolution tracks predicted by the prescription of Hurley et al. (2002) as well as tracks of fully mixed versions of the remnants. We found that our merger remnants have shorter main-sequence lifetimes than predicted by either the Hurley et al. (2002) prescription or the fully mixed models. Our models are also brighter than

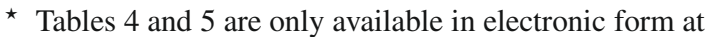
http://www . aanda.org normal stars of the same mass, but not as blue as fully mixed models.

In this work we explore the parameter space for collisions between low-mass main sequence stars relevant for blue straggler formation in old open clusters (such as M 67) and place the findings of Glebbeek et al. (2008) in context. A large grid of stellar collisions covering this parameter space was calculated by Freitag \& Benz (2005). They mainly focussed on collisions with high impact velocity suitable for the galactic centre and their simulations had insufficient resolution to resolve mixing due to the collision. Our work complements theirs by investigating the mixing as well as the long term evolution of the merger remnants. We describe the detailed structure and evolution of the collision products and discuss their dependence on the collision parameters (masses and time of collision) in Sect. 4. We compare the results of our models with observations of blue stragglers in the open clusters M 67 and NGC 188 in Sect. 5. Finally, we show how the global properties of the collision products (luminosity, radius and lifetime) can be described by a simple analytic prescription that can be included in a parametric model of stellar collisions and in $\mathrm{N}$-body simulations (Sect. 6). We discuss our results in Sect. 7.

\section{Initial conditions and parameters of the collisions}

The structure of a merger remnant depends on the structure of the two parent stars, the impact parameter of the collision and the relative velocity at infinity. The structure of the parent stars depends on their masses, chemical composition (characterised by their heavy-element content $Z$ ) and their evolutionary stage. In this work we have restricted ourselves to a section of this 8-dimensional parameter space.

First of all we assume that the two stars involved in the collision are coeval in the sense that both are at the zero-age main 
Table 1. Values used for the different grid parameters. The grid spacing is listed between parentheses. Note that the grids labelled M67 and NGC 188 overlap in part of their age range. We have calculated models for two extra mass ratios for a limited mass range in the M 67 grid to more clearly resolve trends at high and low mass ratios.

\begin{tabular}{lllll}
\hline \hline Name & $Z$ & $t[\mathrm{Myr}]$ & $M\left[M_{\odot}\right]$ & $q$ \\
\hline M 67 & 0.02 & $2800,3100,3400,3700$ & $1.5-2.4(0.1)$ & $0.4-1.0(0.2)$ \\
& & & $1.5-2.0(0.1)$ & $0.5,0.9$ \\
NGC 188 & 0.02 & $3400,3700,4200,4700,5200,5700$ & $1.2,1.3,1.4,1.6,1.8,2.0,2.2$ & $0.4-1.0(0.2)$ \\
GC & 0.001 & $8000-12500(1500)$ & $0.8-1.3(0.1)$ & $0.4-1.0(0.2)$ \\
\hline
\end{tabular}

sequence (ZAMS) at $t=0$ and have the same initial composition, which is a reasonable assumption for stars in clusters. Placing $t=0$ at an earlier stage, e.g. the onset of deuterium burning rather than the ZAMS, will not greatly affect the outcome of our calculations because appreciable composition gradients only build up on the main sequence and we consider a fairly small mass range. We restrict ourselves to collisions between low-mass main sequence stars (by which here we mean that their combined mass does not exceed $2.4 M_{\odot}$, see below) and consider only head-on collisions (i.e., with impact parameter $b=0$ ), in essence meaning that we ignore the effect of rotation in the collision product. This is despite the fact that rotation can be of considerable importance for the structure and evolution of the collision product: even for collisions with a small impact parameter the remnant has sufficient angular momentum that a main sequence star of the same mass would need to rotate faster than its breakup rate (Lombardi et al. 1996; Sills et al. 1997). The physical mechanism and timescale on which the collision products lose their angular momentum are unclear. Methods to incorporate the effect of rotation in a one dimensional stellar evolution code treat rotation as a perturbation to a non-rotating stellar model (Endal \& Sofia 1976; Pinsonneault et al. 1989; Zahn 1992; Heger et al. 2000). These methods are accurate for rotation rates less than about $60 \%$ of the critical (Keplerian) value (Yoon et al. 2004) and it is not clear how to model stars that are even closer to critical rotation. We plan to investigate this problem in future research.

This leaves us with four parameters: the composition, the time of collision $t$ and the masses of the two progenitors. Borrowing nomenclature from the field of binary evolution, we refer to the more massive progenitor as the primary and write its mass as $M_{1}$. Similarly we refer to the less massive progenitor as the secondary and denote its mass by $M_{2}$. With these definitions we can introduce the total initial mass $M=M_{1}+M_{2}$ and the mass ratio $q=M_{2} / M_{1}$. To calculate a grid of models we have used the independent parameters $Z, t, M$ and $q$. We have calculated several grids covering different parts of this parameter space, as listed in Table 1. The grid labelled M 67 covers the parameter space sampled by the $N$-body simulation of Hurley et al. (2005) and is relevant for old open clusters like M67. An extension to the M67 grid is the parameter set labelled NGC 188, which covers the parameter range of interest for the somewhat older open cluster NGC 188. Both these grids use an assumed heavyelement content $Z=0.02$ and an initial hydrogen abundance $X=0.70$. The total initial masses for the collision products were chosen such that the lower limit is just above the present-day turnoff mass and the upper limit is roughly twice the turnoff mass (1.18 $M_{\odot}$ for NGC 188, $1.29 M_{\odot}$ for M 67); see Sect. 5 .

We have also computed a grid (labelled GC) with $Z=0.001$ and $X=0.757$ that is suitable for comparison with globular clusters. In the presentation of our results we will mostly focus on the M 67 and NGC 188 grids but unless otherwise indicated our results apply to the $Z=0.001$ grid as well.

\section{Tools}

The method we use in our study has been described in detail in a separate paper (Glebbeek et al. 2008, hereafter Paper I), so we refer the interested reader to that paper for more details about the procedure of calculating the models and provide just a brief summary here.

For each collision we evolve models of the progenitor stars to the time of collision and then merge the two stars. The structure of the merger remnants is calculated using the Make Me A Star (MMAS) code developed by Lombardi et al. (2002). This code uses the realisation that in low mass stars the quantity

$A=\frac{P}{\rho^{5 / 3}}$

increases outward. The quantity $A$ is closely related to the entropy and the tendency of $A$ to increase reflects the tendency of low entropy material to sink to the centre of the collision product. For this reason the procedure by which the remnant profile is constructed is known as entropy sorting.

Our evolution code is a version of the STARS code developed by Eggleton (1971) and updated by others (Pols et al. 1995). Nuclear reaction rates are from Caughlan \& Fowler (1988) and Caughlan et al. (1985) and we use opacities from Rogers \& Iglesias (1992) and Alexander \& Ferguson (1994). The assumed heavy-element composition is scaled to solar abundances (Anders \& Grevesse 1989). Chemical mixing due to convection (Böhm-Vitense 1958; Eggleton 1972) and thermohaline mixing (Kippenhahn et al. 1980) is taken into account. All models are computed with a mixing-length ratio $l / H_{\mathrm{P}}=2.0$. As in Paper I, we have neglected convective overshooting in all models in this work.

\section{Properties and structure of collision products}

In this section we present the structure and evolution of the merger remnants. We will discuss how the structure of the parent stars affects the structure of the remnant. Details for all our collision models are listed in Table 4 for $Z=0.02$ models and in Table 5 for $Z=0.001$ models. Both these tables are available in the Online version of this paper.

\subsection{Mass loss from the collision}

A fraction $\phi$ of the total mass $M_{1}+M_{2}$ of the progenitor stars is lost during the collision. The fraction $\phi$ is, for central collisions, estimated in MMAS as

$\phi=C \frac{q}{(1+q)^{2}} \frac{R_{1,0.86}+R_{2,0.86}}{R_{1,0.5}+R_{2,0.5}}$,

(Lombardi et al. 2002), where $R_{n, 0.86}$ and $R_{n, 0.5}$ are the radii containing $86 \%$ and $50 \%$ of the mass of parent star $n$ ( 1 or 2 ). The constant $C=0.157$ is determined by calibration to SPH calculations. Typical values of $\phi$ are in the order of a few percent. 
A reasonable approximation over the range of our grid is (see Table 4)

$\phi=0.3 \frac{q}{(1+q)^{2}}$.

Material lost from the stars in the ejecta originates from the envelopes of the parent stars and has the composition of the parent star envelope.

\subsection{Structure of the progenitor stars}

We can distinguish four different collision scenarios between main sequence stars based on the structure of the progenitor stars and the collision product. Low mass main sequence stars ( $\lesssim 1.2 M_{\odot}$ ) have radiative cores while main sequence stars of higher mass have a convective core. This leads to four possible scenarios for a collision:

1. Convective + Convective $\rightarrow$ Convective;

2. Convective + Radiative $\rightarrow$ Convective;

3. Radiative + Radiative $\rightarrow$ Convective;

4. Radiative + Radiative $\rightarrow$ Radiative.

The composition profile of a collision remnant is determined by the entropy profiles of the progenitor stars, which is in turn determined by their masses and their age. If a star has a convective core the material from the core will have a constant entropy, which directly affects the composition profile in the remnant and its subsequent evolution. In a star with a radiative core the entropy increases outward.

The first case will generate remnants with masses $>2.4 M_{\odot}$ and will be the topic of a future paper. The second case occurs in our grid for the higher mass collision products $\left(M>1.7 M_{\odot}\right)$ at extreme mass ratios $(q>0.5)$ and is relevant for clusters where the turnoff mass is larger than $1.2 M_{\odot}$. The third case makes up the majority of the collision products in our grid and is relevant for old open clusters as well as globular clusters. The main difference with case 2 is that in case 2 the core of the primary has an almost flat entropy profile whereas in case 3 the entropy profile is smoother. The final case is only relevant for clusters where the turnoff mass is below $1.2 M_{\odot}$. In younger clusters the collision products of these collisions would be hidden among normal main sequence stars and not stand out as blue stragglers. The progenitor stars are essentially unevolved at the time of collision and the outcome of such a merger is a new unevolved main sequence star.

\subsection{Composition profile}

During a collision material from one parent star is mixed with material of the same entropy from the other star (since it ends up in the same location in the collision product). However, if one of the two stars has a convective core the material from the core can retain its identity because of the nearly flat entropy profile. This can lead to a very steep composition gradient in the remnant.

On the ZAMS lower mass stars have a lower central entropy than higher mass stars (their cores are denser), which means that in a collision between unevolved stars material from the lower mass star will sink to the centre of the collision product. As a result of stellar evolution, the entropy in the core will decrease (the core becomes more compact). Since more massive stars evolve more quickly, the central entropy will decrease more rapidly in massive stars than it will in low mass stars.

Figure 1 shows a number of evolution tracks in the $\log \rho_{\mathrm{c}}-\log T_{\mathrm{c}}$ plane. The location of the ZAMS is indicated by

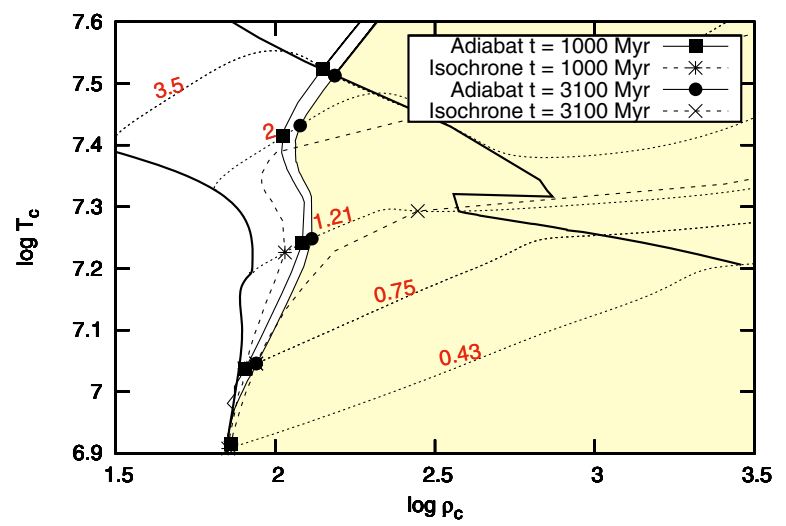

Fig. 1. Evolution tracks of low mass stars in the $\log \rho_{\mathrm{c}}-\log T_{\mathrm{c}}$ plane. Bold lines indicate the locations of the ZAMS (left) and TAMS (right). Dotted lines are evolution tracks, labelled with the ZAMS mass. The solid lines marked with $\mathbf{\square}, \bullet$ are lines of constant entropy across different stellar models, the dashed lines marked with $*, \times$ are isochrones. The shaded region indicates the region where the central entropy is lower than that of a $0.75 M_{\odot}$ star at $3100 \mathrm{Myr}$.

a thick solid line on the left and the location of the terminal age main sequence (TAMS) by a thick solid line on the right. Evolution tracks are dotted and labelled with the mass of the star and two isochrones are shown, one for $1000 \mathrm{Myr}$ and one for 3100 Myr. Two lines of constant entropy across different stellar models are also drawn for different ages. The shaded region indicates the location where the entropy is lower than in the core of a $0.75 M_{\odot}$ star at $t=3100$ Myr. The $t=3100$ Myr isochrone lies almost completely within this region, indicating that in a collision with a more massive star at this age the core of the primary sinks to the centre of the collision product. Conversely, part of the $t=1000 \mathrm{Myr}$ isochrone lies to the left of the line of constant entropy at that age, indicating that in a collision with a more massive star, up to $\approx 1.9 M_{\odot}$, the secondary star will sink to the centre.

For the composition profile in the collision product we can roughly distinguish three cases, which we will denoted by "M", "S" and "P" for "mixed core", "secondary core" and "primary core" respectively. These are illustrated in Fig. 2. The part of the parameter space in the grid for which each of these cases occurs is illustrated in Fig. 3 and listed in Table 4.

\subsubsection{Case $M$}

If the mass difference between the two colliding stars is small (the mass ratio is close to 1 ), the colliding stars have very similar entropy and composition profiles. The composition profile of the collision product will resemble a stretched version of the composition profile in the progenitor stars. This situation is illustrated by the top left panel in Fig. 2. There can be a small molecular weight inversion near the centre if most of the material in the core comes from the secondary star. This case is indicated by $\square$ in Fig. 3. In Fig. 1 this means that the two models are on or close to the line of constant entropy.

\subsubsection{Case $P$}

If the primary is sufficiently evolved that its core entropy is lower than that of the secondary, the core of the primary ends up in the centre of the collision product. In Fig. 1 this means that the primary is in the shaded region of the diagram. In this case the core 


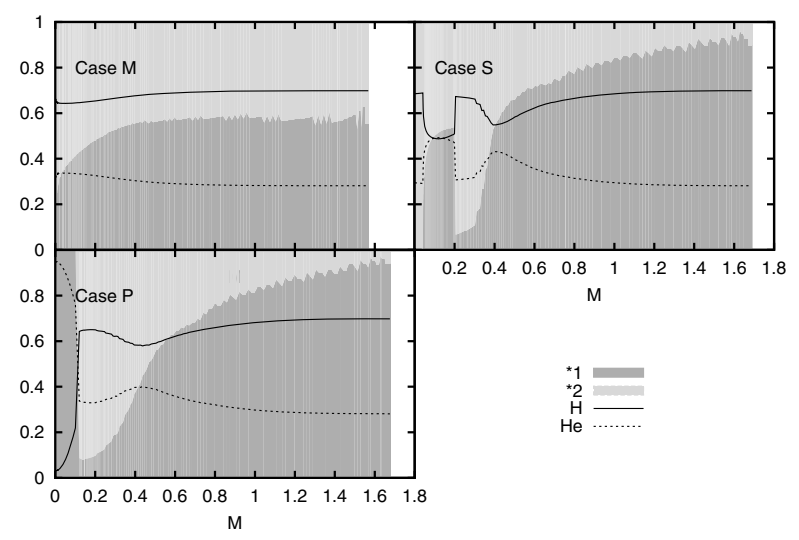

Fig. 2. Diagram showing the distribution of material from the parent stars within three of the collision products: $t=2800, q=0.8, M=1.7$ (corresponding to case $\mathrm{M}$, see text) and $t=2800, q=0.4, M=1.8$ (case $\mathrm{S}$ ) and $t=3700, q=0.4, M=1.8$ (case $\mathrm{P}$ ) for the bottom row. The dark shading indicates the fraction of material from the primary while the lighter region shows the fraction of material from the secondary. Overplotted are the abundances by mass fraction of hydrogen (solid) and helium (dotted).

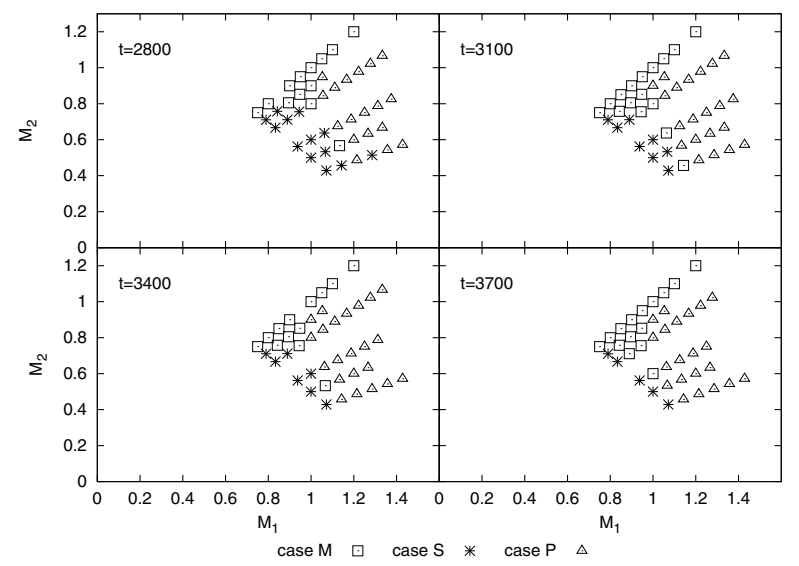

Fig. 3. Illustration of the different cases for the composition profiles in the collision products and the grid parameters for which they occur.

of the merger remnant has a reduced hydrogen content with a very steep increase in hydrogen abundance at its edge and possibly a molecular weight inversion further out as well. A hydrogen burning shell can form at the edge of the core (see Sect. 4.4). This is the situation in the lower left panel of Fig. 2 and is marked in Fig. 3 with $\triangle$. An extreme example of this case occurs when the primary is at the end of the main sequence. The hydrogen depleted core of the primary sinks to the centre of the collision product, producing a new star with a hydrogen depleted core. In other words, collisions involving turnoff stars produce turnoff stars, in agreement with Sills et al. (1997).

\subsubsection{Case S}

If the entropy in the core of the primary is larger than the entropy in the core of the secondary the secondary will sink to the centre of the collision product. In terms of Fig. 1 the primary is to the left of the shaded region. The core of the collision product will be hydrogen-rich, with a helium rich layer on top of it, leading to a pronounced molecular weight inversion. This is the upper left situation in Fig. 2 (a * in Fig. 3). This case will appear for collisions between main sequence stars when the mass

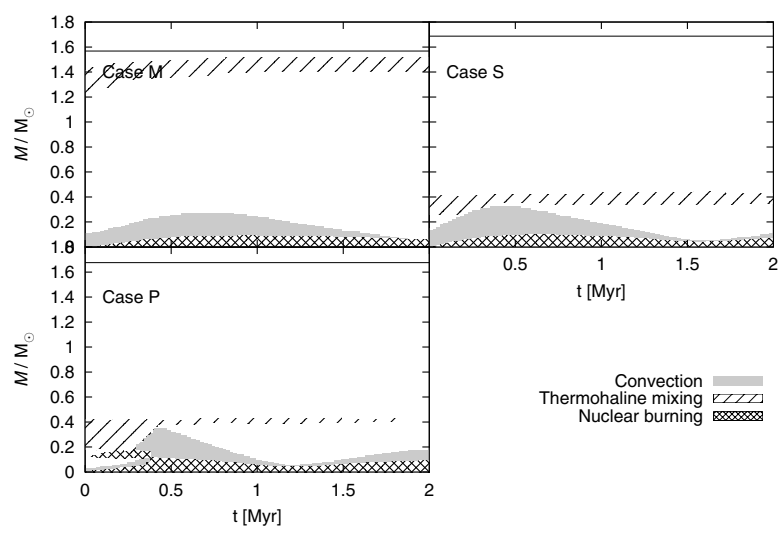

Fig. 4. Kippenhahn diagrams showing the mixing regions during the contraction phase for the same collision products as shown in Fig. 2.

ratio of the colliding stars is small $(\sim 0.4)$. If the mass ratio is even smaller $(\lessgtr 0.2)$, this will happen even after the primary has already evolved off the main sequence.

\subsection{Reignition of hydrogen and mixing}

Initially there is little or no nuclear burning in the core of the collision products and nuclear burning is unimportant as an energy source during the contraction to the main sequence.

Because the core of the collision product has the entropy of a lower mass star it lies on a lower adiabat than the core of a main sequence star of the same mass and composition. In practice, this means that the collision product is initially to the right of its main sequence position in the $\rho_{\mathrm{c}}-T_{\mathrm{c}}$ plane, at higher central densities. In order for the star to come into thermal equilibrium the entropy in the core needs to increase. The core will first expand and then heat up (see Paper I). Once hydrogen burning takes over as the main energy source the evolution of the collision product proceeds analogously to that of a normal main sequence star but with an abnormal composition profile (again see Paper I).

If there is a steep composition gradient at the edge of the core (when one of the two stars had a convective core), it is possible for the nuclear energy generation rate to peak at this location due to the composition dependence of the reaction rate. In effect, hydrogen will burn in the core as well as in a shell outside the core. This hydrogen burning shell is a transient feature that is destroyed when mixing reduces the steepness of the composition gradient. The hydrogen burning shell is important because it can drive a convection zone that mixes the layers above it. Examples of the mixing regions during the contraction phase in each of the three cases in Fig. 2 are shown in Fig. 4.

Because the material outside the core can be hydrogen-rich, convection can bring extra hydrogen to the core, effectively rejuvenating the star. Table 4 gives the central hydrogen abundace immediately after the collision and at the beginning of the main sequence phace. How much hydrogen is mixed into the core depends on mixing processes in the layer outside the core.

Helium-rich material on top of a layer of hydrogen-rich material causes a molecular weight inversion, which gives rise to thermohaline mixing (Kippenhahn et al. 1980). The material with higher molecular weight is supported by thermal buoyancy. Diffusion of heat from the material causes it to lose buoyancy and sink due to its higher mean molecular weight. The mixing continues until the molecular weight inversion is removed. Thermohaline mixing is important in the collision products during the contraction phase in the cases where material from the 


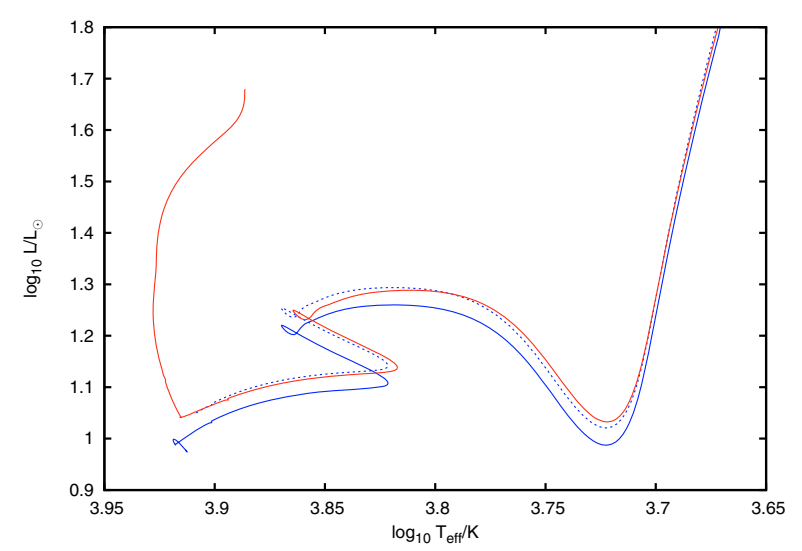

Fig. 5. Evolution track of the $t=2800, q=0.8, M=1.9$ remnant with a mass of $1.75 M_{\odot}$ (top solid line) compared with the evolution track of a normal star of the same mass (bottom solid line). The shape of the evolution tracks after the contraction of the merger remnant is very similar, but the merger track is offset to higher luminosity and is slightly cooler at the end of the main sequence. The dashed line is a shifted version of the main sequence track according to Eqs. (13) and (19), see Sect. 6 .

core of the primary overlies material from the secondary, which happens for cases $\mathrm{P}$ and $\mathrm{S}$, discussed in Sect. 4.3 and shown in Fig. 4. By the time the collision products reach the main sequence the molecular weight inversion has been smoothed out. As a result more helium has been mixed into the centre of the collision product than would have been the case if thermohaline mixing had been ignored. This reduces the amount of hydrogen available for nuclear burning and decreases the lifetime of the collision product.

A hydrogen burning shell at the edge of the core can drive a convection zone that mixes in material from the layers above. This material can be either hydrogen-rich or helium rich, depending on the particular case. The convection zone can connect to the thermohaline layer, which means extra helium is mixed into the inner region of the star.

When the convective core is formed it can in turn connect to the convection zone driven by the burning shell (Fig. 4, lower left panel). If there is no burning shell, it can connect to the thermohaline layer (Fig. 4, upper left panel). In either case the end result is that the interior of the collision product is mixed below the molecular weight inversion. This produces a large central region that has been enhanced in helium and is larger than the convective core itself. The net effect is comparable to that of a star in which the convective core was larger initially but has shrunk.

Outside the mixed region, the composition profile is shallower than in a normal star of the same mass. This affects the structure of the star most strongly during the main sequence although it continues to affect the star during later evolutionary stages.

\subsection{Main sequence evolution}

Once the merger remnant has attained thermal equilibrium its further evolution proceeds similarly to that of a normal star of the same mass. In particular, the shape of the evolution track in a Hertzsprung-Russell diagram is the same. The composition profile modifies the evolution mainly in two ways: by affecting the lifetime and by affecting the luminosity. A typical evolution track is shown in Fig. 5.
The luminosity is affected by the higher helium content of the envelope. This affects the structure of the star through the equation of state (due to the mean molecular weight) and the opacity, as discussed in Paper I, and results in a larger radius and higher luminosity. Because the surface layers do not show helium enhancement the effective temperature is not strongly affected, although there is a systematic trend that the coolest part of the track (just before hydrogen exhaustion at the end of the main sequence) is shifted to slightly lower temperatures. Shifts in the luminosity and effective temperature are listed in Table 4 for all our merger remnants.

The remaining lifetime of the star is reduced by the higher luminosity, but also by the central hydrogen abundance which is lower than the abundance in the envelope. Because of this the merger remnants do not resemble zero-age main sequence (ZAMS) stars after they settle down and are both redder and brighter than ZAMS stars of the same mass.

While on the Hertzsprung gap tracks of the merger remnants are still brighter than tracks of normal stars of the same mass. On the giant branch the difference between the two tracks vanishes. When the first dredge-up occurs the convective envelope enters the layer where the composition had been modified by the merger. This enhances the post-dredge-up abundances of helium and nitrogen and reduces the abundance of carbon compared to a normal red giant (see Paper I for more details).

\subsection{The effect of lower metallicity}

The heavy-element abundance $Z$ affects the structure and evolution of stars. In general stars with a lower $Z$ are hotter, more luminous and more compact than stars of the same mass at higher $Z$. The mass at which a convective core develops is higher and the lifetime is reduced.

This changes the structure of merger remnants in subtle ways. For instance, the relative rate at which the progenitor stars evolve along the main sequence is different for $Z=0.02$ and $Z=0.001$. This means that if we consider a $Z=0.001$ collision at a time when the primary has passed the same fraction of its main sequence lifetime, the secondary will not have spent the same fraction of its main sequence lifetime as in the $Z=0.02$ collision. Qualitatively the description given in the previous subsections remains valid for smaller $Z$. The data for the merger remnants calculated for our grid are listed in Table 5.

The mass loss from the collisions is slightly lower than for the $Z=0.02$ grid although $C=0.3$ is still a reasonable guess. The reason for this can be seen from (2): the stars at lower $Z$ are more centrally condensed than at higher $Z$ and the ratio $\left(R_{1,0.86}+R_{2,0.86}\right) /\left(R_{1,0.5}+R_{2,0.5}\right)$ is smaller.

\section{HRD distributions: comparison with M 67 and NGC 188}

In this section we compare the predicted locations of the collision products from our grid with the observed blue stragglers in the open clusters M 67 and NGC 188. For both of these clusters Pols et al. (1998) determined isochrone fits. For consistency we employ the same cluster parameters they used, as listed in Table 2.

In order to make the comparison between our models and the observations it is necessary to convert the theoretical surface parameters $\left(L, T_{\text {eff }}, \log g\right)$ to the observable magnitude and colour $\left(M_{V}, B-V\right)$. This conversion is done using the atmosphere 
Table 2. Ages determined from isochrone fitting for adopted values of distance modulus and reddening. See Pols et al. (1998) for details.

\begin{tabular}{llllll}
\hline \hline Name & $\log _{10}$ age $/ \mathrm{yr}$ & $M_{\text {to }}$ & {$[\mathrm{Fe} / \mathrm{H}]$} & $m-M$ & $E(B-V)$ \\
\hline M 67 & 9.60 & 1.29 & -0.06 & 9.60 & 0.032 \\
NGC 188 & 9.76 & 1.18 & -0.06 & 11.40 & 0.12 \\
\hline
\end{tabular}

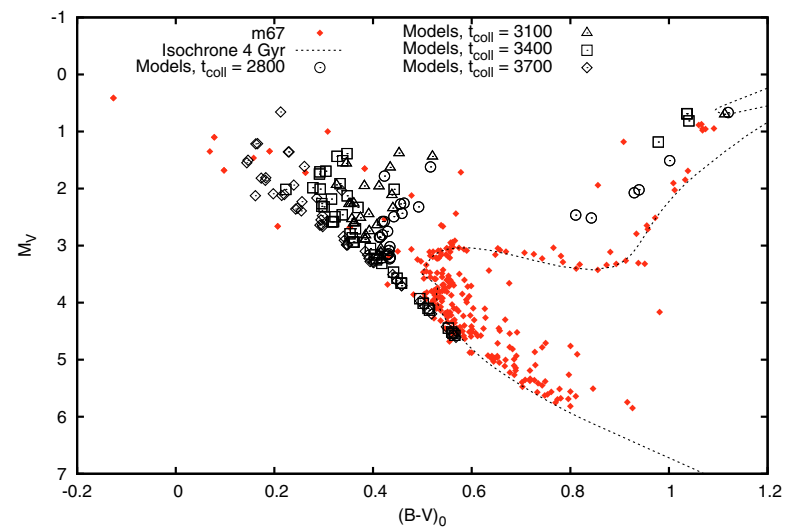

Fig. 6. Observed colour-magnitude diagram of the open cluster M67, with blue stragglers highlighted and the positions of the stars in our collision grid at the age of M 67 overplotted. Different symbols correspond to different times of collision, as indicated.

models from Kurucz (1992) with empirical corrections from Lejeune et al. (1997).

Figure 6 shows the observed colour-magnitude diagram of M $67(\bullet)$. The $V$ and $B-V$ data is taken from Sandquist (2004) supplemented with Montgomery et al. (1993) for blue stragglers that were missing from the Sandquist (2004) data. Membership information (based on proper motions) is taken from Sanders (1977). Sandquist (2004) gives a distance modulus $m-M=9.60$ and reddening $E(B-V)=0.03$, consistent with the values used by Pols et al. (1998) to construct the $4 \mathrm{Gyr}$ isochrone. The location of the collision remnants at $4 \mathrm{Gyr}$ are plotted with different symbols, corresponding to the different times of collision. It is important to stress that our model is not a population synthesis model, so one should not draw conclusions from the density of the model points.

Our models lie in the observed blue straggler region. Some of the lower-mass merger remnants with masses below the turnoff appear among the normal main-sequence stars. The bluest blue straggler (S977 with $M_{V}=0.413$ and $B-V=-0.126$, Mathys 1991 ) is clearly outside the region covered by our models, but it is thought to have a mass larger than twice the turnoff mass (Sandquist 2005) and therefore cannot be formed by a single collision event. A few other blue stragglers are somewhat bluer than the region covered by our models. They are apparently single stars and are thus very likely to be the result of a stellar merger. Their blue positions can be explained if they have undergone much stronger internal mixing than our models, or if they have been formed more recently and are in that sense younger than our models. These blue stragglers are all slow rotators $\left(v \sin i \leq 120 \mathrm{~km} \mathrm{~s}^{-1}\right)$ compared to normal stars of the same spectral type (Mathys 1991), suggesting that rotational mixing has not played a major role in their evolution. We therefore prefer the second explanation, which suggests that M 67 is dynamically active, in other words, collisional blue stragglers are still being formed. This conclusion is consistent with the $N$-body model of Hurley et al. (2005).

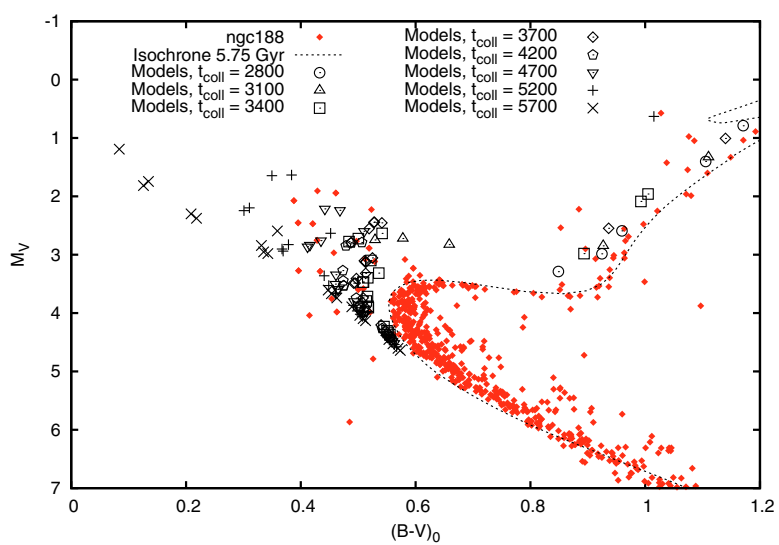

Fig. 7. As Fig. 6 for NGC 188.

A number of our merger remnants have evolved beyond the end of the main sequence and appear on the giant branch but to the blue of the normal cluster giant branch, indicating that merger remnants can still stand out in the CMD even after the main sequence has ended. Two stars in M67 are located in this region (S1040 and S1273); both are spectroscopic binaries as well as unusual X-ray sources (van den Berg et al. 1999). It is not clear whether these stars are merger remnants, their unusual location may also be the result of a superposition of a giant and a turnoff star. In the case of S1040 the companion is probably a white dwarf and the orbit is circular (Mathieu et al. 1990), so the system has likely undergone mass transfer.

Figure 7 shows the comparison with NGC 188, with $V, B-V$ and membership probabilities taken from Platais et al. (2003). Although NGC 188 is known to be older than M67, different authors give different age estimates (von Hippel \& Sarajedini 1998; Platais et al. 2003; Carraro et al. 1994). We adopt $m-M=$ 11.4 and $E(B-V)=0.12$ and an age of $5.8 \mathrm{Gyr}$, as in Pols et al. (1998). By contrast, Bonatto et al. (2005) find $m-M=11.1$ and $E(B-V)=0.0$ from fitting a $t=7$ Gyr Padova isochrone.

Our models fill a larger region than the observed blue straggler region, indicating that NGC 188 has not formed massive blue stragglers recently. This suggests that it is less dynamically active than M67. As for M 67 we note that a number of merger remnants are beyond the main sequence and on the giant branch, again parallel to the cluster giant branch.

\section{Analytical description of results}

To quantify the results of our collision calculations and compare the evolution tracks of the collision products with evolution tracks of normal single stars it is necessary to specify which models should be compared. In particular, we need to define an equivalent main sequence star model.

Apart from the mass $M$ of the collision product the remaining lifetime is a good parameter to consider. For this reason it is convenient to introduce the fractional age $f$, which is the age of a star expressed in units of its main sequence lifetime,

$f=\frac{t}{\tau_{\mathrm{MS}}}$

where $t$ is the absolute age and $\tau_{\mathrm{MS}}=\tau_{\mathrm{MS}}(M, Z)$ is the main sequence lifetime of a star of mass $M$ and composition specified by $Z$, which is listed in Table 4 for the stars in our grid. As long as the star is on the main sequence, $0<f<1$. We define the apparent age $f_{\text {app }}$ of the collision product as the fractional age of a 
normal main sequence star with the same remaining lifetime $t_{\mathrm{MS}}$. These two quantities are related by

$t_{\mathrm{MS}}=\tau_{\mathrm{MS}}\left(1-f_{\mathrm{app}}\right)$.

In practice, we know $t_{\mathrm{MS}}$ from the detailed models (see Table 4) for the collision products and we can thus determine $f_{\text {app }}$. If we can predict $f_{\text {app }}$ from the global properties of the parent stars we can predict the collision product lifetime. In the following we will describe a formalism that allows us to do this using only standard stellar models and we give a set of transformation rules that can be used to transform a standard stellar evolution track to approximate the track of a merger remnant. By a standard evolution track we mean the evolution track of a single star that is evolved from the zero-age main sequence. For the standard tracks one can use the Hurley et al. (2000) recipe, or interpolate on a grid of single star models. Our recipe can be used to improve the accuracy of evolution tracks for merger remnants in star cluster simulations.

\subsection{Collision product lifetimes}

In normal main sequence stars there is a unique relation between the amount of hydrogen and the age of the star. Collision products show a similar relation. Although it might seem better to relate the age of the star to the amount of hydrogen in the core rather than the total amount of hydrogen, in practice this is not a good measure of the age of the star for collision products because mixing as described in Sect. 4.4 can increase the central hydrogen abundance compared to its post-collision value.

The relation between the amount of hydrogen and the age of the star is the starting point for a relation between the global properties of the parent stars and the apparent age $f_{\text {app }}$ of the collisions product. Let's consider a simple stellar model that divides the star in two parts: a core where hydrogen is burned to helium and an envelope which is not affected by nuclear burning on the main sequence. The core has a mass fraction $q_{\mathrm{c}}$ of the total mass $M$. Such a model is not really applicable to low-mass stars, which do not have a well-defined core. However, we can generalise the model by defining $q_{\mathrm{c}}$ to be the fraction of hydrogen that is burned during the main sequence, so that

$q_{\mathrm{c}}=\frac{X_{0}-\langle X\rangle_{\mathrm{TAMS}}}{X_{0}}$,

with $X_{0}$ the initial (ZAMS) hydrogen fraction. We can think of $q_{\mathrm{c}}$ as being the effective core mass fraction. If we approximate the nuclear burning rate as steady, the average (total) amount of hydrogen follows from the relative age $f$ as

$\langle X\rangle(t)=X_{0}\left(1-q_{\mathrm{c}} f\right)$.

In practice, $q_{\mathrm{c}}$ will depend on the stellar mass $M$ and composition $Z$ and needs to be found from detailed stellar models. It is convenient to have an analytic approximation for $q_{\mathrm{c}}(M)$. We use the rational function

$q_{\mathrm{c}}(M)=\frac{1+c_{1} M+c_{2} M^{3}+c_{3} M^{5}+c_{4} M^{7}}{c_{5}+c_{6} M^{2}+c_{7} M^{4}+c_{8} M^{6}}$

$\left(M\right.$ in $\left.M_{\odot}\right)$.

The coefficients $c_{i}$ have been found by least-squares fitting to detailed models calculated with the STARS code for masses in the range $M=0.4 \ldots 75 M_{\odot}$. These models were computed without convective overshooting. The fitting coefficients are listed in Table 3. In the mass range $0.4 \ldots 4.0 M_{\odot}$ a somewhat more accurate fit is possible without the terms involving $M^{6}$ and $M^{7}$
Table 3. Coefficients for the fitting formula (8) for $q_{\mathrm{c}}$. The mass range and metallicity for which the coefficients in each column are valid are given in the top row. The bottom row (rms) gives the root mean square error of the fits.

\begin{tabular}{lllll}
\hline \hline & $Z=0.02$ & & $Z=0.001$ & \\
$c$ & $0.4-75 M_{\odot}$ & $0.4-4 M_{\odot}$ & $0.4-75 M_{\odot}$ & $0.4-5 M_{\odot}$ \\
\hline$c_{1}$ & -0.685213 & -0.999710 & -0.682671 & -0.873596 \\
$c_{2}$ & 0.289269 & 0.276843 & 0.185036 & 0.165455 \\
$c_{3}$ & 0.0123223 & 0.0393513 & 0.00658021 & 0.0158297 \\
$c_{4}$ & $3.3357 \mathrm{e}-6$ & 0 & $7.5450 \mathrm{e}-7$ & 0 \\
$c_{5}$ & 2.70964 & 2.75579 & 2.71437 & 2.72675 \\
$c_{6}$ & 1.44963 & -1.70434 & 0.334367 & -1.33733 \\
$c_{7}$ & 0.629121 & 1.46625 & 0.343889 & 0.644498 \\
$c_{8}$ & 0.000493117 & 0 & 0.000185160 & 0 \\
$\mathrm{rms}$ & $1.4 \%$ & $1.2 \%$ & $1.7 \%$ & $1.3 \%$ \\
\hline
\end{tabular}

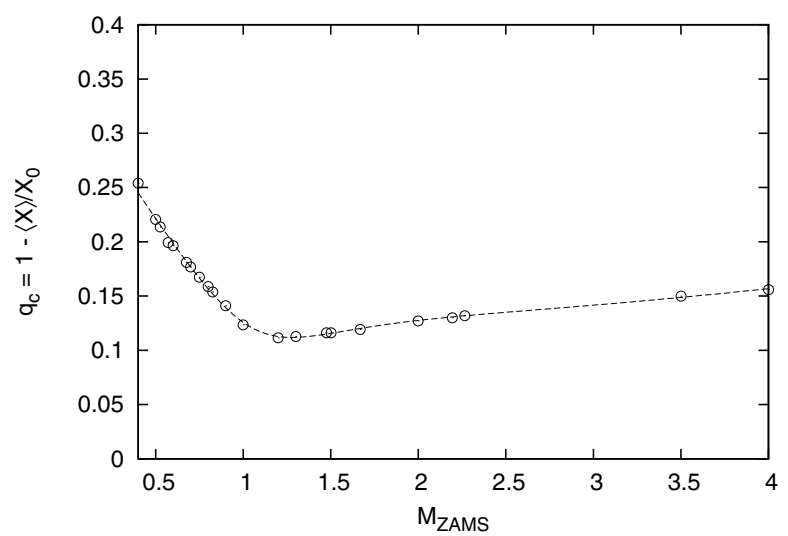

Fig. 8. Effective core mass fraction for main sequence stars against ZAMS mass, for $Z=0.02(\odot)$. The fitting formula (8) in the mass range $0.4-4.0 M_{\odot}$ is shown as a dashed line.

(second and fourth column in Table 3); the quality of this fit for $Z=0.02$ can be checked against Fig. 8. It is of course also possible to interpolate on a table of detailed models.

To find an expression for $f_{\text {app }}$ we first need to know the amount of hydrogen in the collision product. To do this correctly we should consider the material that is ejected during the collision. This comes from the progenitor star envelopes and therefore has the initial ZAMS hydrogen abundance $X_{0}$. If $\phi$ is the fraction of mass lost during the collision (see Sect. 4.1) then the mass $M$ of the collision product is $M=(1-\phi)\left(M_{1}+M_{2}\right)$. The average hydrogen abundance $\langle X\rangle$ in the collision product immediately after the collision is then given by

$M\langle X\rangle=M_{1}\langle X\rangle_{1}(t)+M_{2}\langle X\rangle_{2}(t)-\phi\left(M_{1}+M_{2}\right) X_{0}$.

Inserting (7) for the hydrogen abundance in the parent stars and solving for $\langle X\rangle$,

$\langle X\rangle=X_{0}\left(1-\frac{1}{1-\phi} \frac{q_{\mathrm{c}, 1} f_{1}+q_{\mathrm{c}, 2} f_{2} q}{1+q}\right)$.

The equivalent age $f_{\text {app }}$ is determined by the amount of hydrogen $q_{\mathrm{M}}$ that gets mixed into the burning region through

$\langle X\rangle=X_{0}\left(1-q_{\mathrm{M}} f_{\mathrm{app}}\right)$.

As already mentioned in Sect. 4.4 the larger fraction of processed material within the merger remnant has an effect that is comparable to that of a star with an initially larger core mass. Setting $q_{\mathrm{M}}=\alpha q_{\mathrm{c}}(M)$, we find $f_{\text {app }}$ from (10) as

$f_{\mathrm{app}}=\frac{1}{\alpha q_{\mathrm{c}}(M)} \frac{1}{1-\phi} \frac{q_{\mathrm{c}, 1} f_{1}+q_{\mathrm{c}, 2} f_{1} q}{1+q}$. 


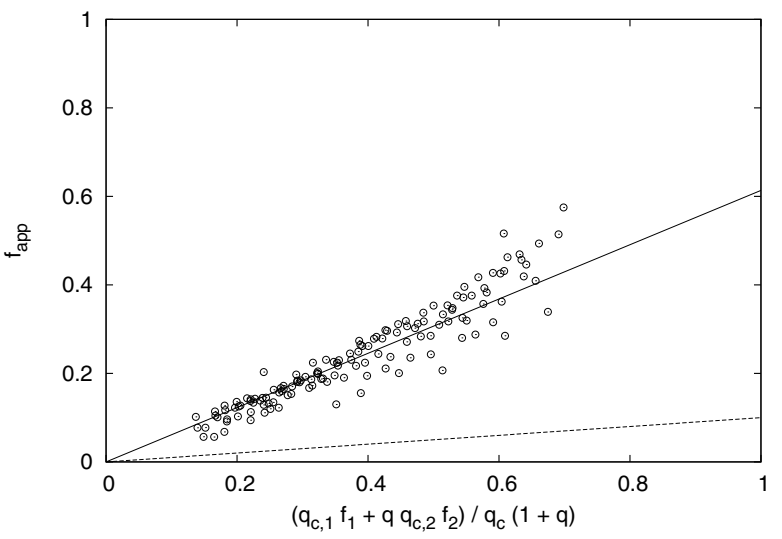

Fig. 9. The predicted apparent age according to (12), for $\alpha=1.67$ (solid line). The detailed models are indicated by $\odot$. The dashed line is the prescription from Hurley et al. (2000).

The parameter $\alpha$ parametrises the amount of mixing during the collision and settling to the main sequence, with $\alpha=1$ meaning no mixing at all and $\alpha=1 / q_{\mathrm{c}}(M)$ meaning homogeneous mixing. Our expression (12) is a generalisation of Eqs. (69) in Tout et al. (1997) and (80) in Hurley et al. (2002), which are recovered in the special case $\phi=0, q_{\mathrm{c}, 1}=q_{\mathrm{c}, 2}=q_{\mathrm{c}}(M)=1 / 10$ and $\alpha=1 / q_{\mathrm{c}}(M)$.

The parameter $\alpha$ can be determined from the models in our grid because all other factors in Eq. (12) are known. In principle it should depend on the evolutionary stages of the two stars involved in the collision, but in practice the value $\alpha=1.67$ works well for all models in our $Z=0.02$ grid, while $\alpha=1.43$ works well for our $Z=0.001$ grid.

Figure 9 compares the predicted lifetime according to (12) to the lifetime from our detailed models. Overall agreement is very good, although the detailed models show more scatter. The dashed line is the prescription from Hurley et al. (2000), which predicts much longer lifetimes, especially for collisions involving very evolved stars. This increases the predicted number of observable blue stragglers formed through collisions by up to a factor 2 compared to our detailed models.

\subsection{Collision product luminosities}

The luminosity of the collision products follows very well from the homology relation (Kippenhahn \& Weigert 1990)

$L_{\text {merger }}=L_{\mathrm{MS}}\left(\frac{\mu_{\mathrm{merger}}}{\mu_{\mathrm{MS}}}\right)^{4}$,

here, $\mu_{\text {merger }}$ is the average mean molecular weight in the collision product at the start of the main sequence, which is the same as the average mean molecular weight after the collision because the contraction phase is fast enough that composition changes due to nuclear reactions can be ignored. The average mean molecular weight of the main sequence model $\mu_{\mathrm{MS}}$ needs to be chosen at the equivalent stage of evolution. Because the shift in effective temperature is small we can choose the point in the evolution track with the same $T_{\text {eff }}$ as the equivalent point. The averages of the mean molecular weight need to be calculated with respect to the mass (as opposed to the radius), which is the same as averaging over all particles.

It is possible to get an estimate for the mean molecular weight using the simple model (7). For a fully ionised hydrogen/helium mixture, the mean molecular weight can be approximated by (e.g. Weiss et al. 2004)

$\mu^{-1}=2 X+\frac{3}{4} Y+\frac{1}{2} Z$,

or, by eliminating the helium abundance $Y$,

$\mu^{-1}=\frac{1}{4}(5 X+3-Z)$.

Then

$\frac{\mu_{\text {merger }}}{\mu_{\mathrm{MS}}}=\frac{5\langle X\rangle_{\mathrm{MS}}+3-Z}{5\langle X\rangle_{\mathrm{merger}}+3-Z}$.

For the main sequence star we can use (7) directly but to match the hydrogen abundance of the merger remnant we use a modified version of (7),

$\langle X\rangle_{\text {merger }}=X_{0}\left(1-\beta-\alpha q_{\mathrm{c}} f_{\text {app }}\right)$.

Physically, this extra parameter is necessary because $f_{\text {app }}$ was calibrated to the main sequence timescale $\tau_{\mathrm{MS}}$ for a normal star. The collision products behave more like stars with a different initial composition and can have a different main sequence timescale. In other words, $f_{\text {app }}$ does not represent the evolutionary stage of the collision product, just its remaining lifetime. Mathematically, the need for an extra parameter arises because we are now fitting two quantities (the lifetime and the hydrogen abundance).

The offset should vanish if the progenitor stars were ZAMS stars (in which case $f_{\text {app }}=0$ ) or if there was no mixing during the collision $(\alpha=1)$. This is satisfied if $\beta \propto(\alpha-1) f_{\text {app }}$. We find that setting $\beta=(\alpha-1) q_{\mathrm{c}} f_{\mathrm{app}} /\left(1-q_{\mathrm{c}}\right)$ gives a good match for all collisions in our grid. Inserting this expression in (17) gives

$\langle X\rangle_{\text {merger }}=X_{0}\left(1-\alpha q_{\mathrm{c}} \frac{\alpha\left(2-q_{\mathrm{c}}\right)-1}{\alpha\left(1-q_{\mathrm{c}}\right)} f_{\mathrm{app}}\right)$.

Setting $\delta\langle X\rangle=\langle X\rangle_{\mathrm{merger}}-\langle X\rangle_{\mathrm{MS}}$ and introducing $\mu_{0}=5\langle X\rangle_{\mathrm{MS}}+$ $3-Z$,

$\frac{\mu_{\text {merger }}}{\mu_{\mathrm{MS}}}=\frac{\mu_{0}}{\mu_{0}+5 \delta\langle X\rangle} \approx 1-5 \frac{\delta\langle X\rangle}{\mu_{0}}$.

From (7) and (11),

$\delta\langle X\rangle=X_{0} q_{c} f_{\text {app }} \frac{\left(2-q_{\mathrm{c}}\right)(1-\alpha)}{1-q_{\mathrm{c}}}$.

Physically, $\alpha>1$ and we see that in that case $\langle\mu\rangle_{\text {merger }}>\langle\mu\rangle_{\mathrm{MS}}$, as we would expect.

The luminosity shift predicted by our analytic prescription is shown in Fig. 5 (dashed line). The main sequence part of the track is very well represented by our prescription, although the hook at the end is slightly too hot. Even the Hertzsprung gap and the giant branch are very well matched with the same shift.

\subsection{Collision product effective temperatures and radii}

The effective temperature of the merger remnants is not very different from that in a normal star of the same mass (see the listed temperature shifts in Table 4), but the reddest point of the main sequence hook is slightly cooler. 
Since the effective temperature of the collision products is very similar to that of normal stars it is possible to use the effective temperature expected for normal stars. Taking the luminosity from (13) the radius of the collision product follows from the Stefan-Boltzmann law, so that

$R_{\text {merger }}=R_{\mathrm{MS}}\left(\frac{\mu_{\mathrm{merger}}}{\mu_{\mathrm{MS}}}\right)^{2}$.

\section{Discussion and conclusions}

We have calculated a grid of stellar evolution models for the remnants of collisions between main sequence stars. These models improve on the models in the literature by using a better model for the initial structure of the merger remnant (Bailyn \& Pinsonneault 1995) or by studying a larger portion of the $t, q, M$ parameter space (Sills et al. 1997).

We have compared the position of our models in the Hertzsprung-Russell diagram with the observed blue straggler regions in the open clusters M 67 and NGC 188 and found that our models can populate the blue straggler region, provided that the blue stragglers in M 67 are relatively "young" and those in NGC 188 are relatively "old".

We have used our results to formulate a recipe that can be used to transform a normal evolution track, which can be obtained from fitting formulae (Hurley et al. 2000) or by interpolation on a table (e.g. Pols et al. 1998; Girardi et al. 2002). This can be used to improve the treatment of collisions in $\mathrm{N}$-body calculations and population modelling.

Our models do not include the effect of rotation. Rotation can modify our results in two ways: collision products need to lose angular momentum and therefore mass to reach hydrostatic equilibrium and rapid rotation can induce mixing. It is clear that for a collision product to survive angular momentum must be lost but the mechanism by which this occurs is unclear. Magnetic fields may be involved, but their role is not well understood. Rotational mixing can enrich the stellar envelope in helium, increasing the luminosity and extending the stellar lifetime. By ignoring rotation we have assumed that an efficient mechanism to remove the angular momentum operates that spins the star down sufficiently that rotational mixing is not important for the long-term evolution. The fact that blue stragglers in M67 are observed to be rotating slowly (Mathys 1991) can be interpreted as an indication that such an efficient mechanism for removing angular momentum does operate in reality.

Although our models can fill the observed blue straggler region in M67 and NGC 188, this does not directly give information about the expected distribution of blue stragglers in the colour magnitude diagram because the models need to be weighted with the probability for each collision. This requires a dynamical (or statistical, e.g. Monte-Carlo) model for the cluster evolution.

There are several directions for future research that could be explored. From the side of cluster modelling it is interesting to study the effect of our predicted corrections to the evolution tracks on the number of expected blue stragglers and on the blue straggler luminosity function. On the topic of detailed modelling of merger remnant evolution, including rotation and rotational mixing (extending the work of Sills et al. 2001, 2005) is a logical next step. However, this needs to be coupled with a mechanism for angular momentum loss from the merger remnant, which requires a better understanding of mass loss from rapidly rotating low mass stars, possibly involving magnetic fields as well.
A simple equilibrium dynamo may not be adequate to model the magnetic field of a merger remnant, which might be very different from the magnetic field of a normal star following the collision. Magnetohydrodynamic models of stellar mergers and stellar evolution models including magnetic fields are also relevant in this light and are a logical next step.

Acknowledgements. We thank the referee, Alison Sills, for valuable comments and Frank Verbunt for useful discussion on the comparison with observations of M 67. E.G. is supported by NWO under grant 614.000.303.

\section{References}

Alexander, D. R., \& Ferguson, J. W. 1994, ApJ, 437, 879

Anders, E., \& Grevesse, N. 1989, Geochim. Cosmochim. Acta, 53, 197

Bailyn, C. D., \& Pinsonneault, M. H. 1995, ApJ, 439, 705

Böhm-Vitense, E. 1958, ZsAp, 46, 108

Bonatto, C., Bica, E., \& Santos, Jr., J. F. C. 2005, A\&A, 433, 917

Carraro, G., Chiosi, C., Bressan, A., \& Bertelli, G. 1994, A\&AS, 103, 375

Caughlan, G. R., \& Fowler, W. A. 1988, Atomic Data and Nuclear Data Tables, 40,283

Caughlan, G. R., Fowler, W. A., Harris, M. J., \& Zimmerman, B. A. 1985, Atomic Data and Nuclear Data Tables, 32, 197

Eggleton, P. P. 1971, MNRAS, 151, 351

Eggleton, P. P. 1972, MNRAS, 156, 361

Endal, A. S., \& Sofia, S. 1976, ApJ, 210, 184

Freitag, M., \& Benz, W. 2005, MNRAS, 358, 1133

Girardi, L., Bertelli, G., Bressan, A., et al. 2002, A\&A, 391, 195

Glebbeek, E., Pols, O. R., \& Hurley, J. R. 2008, A\&A, 488, 1007

Heger, A., Langer, N., \& Woosley, S. E. 2000, ApJ, 528, 368

Hurley, J. R., Pols, O. R., \& Tout, C. A. 2000, MNRAS, 315, 543

Hurley, J. R., Tout, C. A., Aarseth, S. J., \& Pols, O. R. 2001, MNRAS, 323, 630

Hurley, J. R., Tout, C. A., \& Pols, O. R. 2002, MNRAS, 329, 897

Hurley, J. R., Pols, O. R., Aarseth, S. J., \& Tout, C. A. 2005, MNRAS, 363, 293

Kippenhahn, R., Ruschenplatt, G., \& Thomas, H.-C. 1980, A\&A, 91, 175

Kippenhahn, R., \& Weigert, A. 1990, Stellar Structure and Evolution, XVI, 468 (Berlin, Heidelberg, New York: Springer-Verlag), also Astronomy and Astrophysics Library

Kurucz, R. L. 1992, in The Stellar Populations of Galaxies, ed. B. Barbuy, \& A. Renzini, IAU Symp., 149, 225

Lejeune, T., Cuisinier, F., \& Buser, R. 1997, A\&AS, 125, 229

Lombardi, Jr., J. C., Rasio, F. A., \& Shapiro, S. L. 1996, ApJ, 468, 797

Lombardi, Jr., J. C., Warren, J. S., Rasio, F. A., Sills, A., \& Warren, A. R. 2002, ApJ, 568, 939

Mathieu, R. D., Latham, D. W., \& Griffin, R. F. 1990, AJ, 100, 1859

Mathys, G. 1991, A\&A, 245, 467

Montgomery, K. A., Marschall, L. A., \& Janes, K. A. 1993, AJ, 106, 181

Pinsonneault, M. H., Kawaler, S. D., Sofia, S., \& Demarque, P. 1989, ApJ, 338, 424

Platais, I., Kozhurina-Platais, V., Mathieu, R. D., Girard, T. M., \& van Altena, W. F. 2003, AJ, 126, 2922

Pols, O. R., Tout, C. A., Eggleton, P. P., \& Han, Z. 1995, MNRAS, 274, 964

Pols, O. R., Schroder, K.-P., Hurley, J. R., Tout, C. A., \& Eggleton, P. P. 1998, MNRAS, 298, 525

Portegies Zwart, S. F., Makino, J., McMillan, S. L. W., \& Hut, P. 1999, A\&A, 348,117

Rogers, F. J., \& Iglesias, C. A. 1992, ApJS, 79, 507

Sanders, W. L. 1977, A\&AS, 27, 89

Sandquist, E. L. 2004, MNRAS, 347, 101

Sandquist, E. L. 2005, ApJ, 635, L73

Sills, A., Lombardi, Jr., J. C., Bailyn, C. D., et al. 1997, ApJ, 487, 290

Sills, A., Faber, J. A., Lombardi, Jr., J. C., Rasio, F. A., \& Warren, A. R. 2001, ApJ, 548, 323

Sills, A., Adams, T., \& Davies, M. B. 2005, MNRAS, 358, 716

Tout, C. A., Aarseth, S. J., Pols, O. R., \& Eggleton, P. P. 1997, MNRAS, 291, 732

van den Berg, M., Verbunt, F., \& Mathieu, R. D. 1999, A\&A, 347, 866

von Hippel, T., \& Sarajedini, A. 1998, AJ, 116, 1789

Weiss, A., Hillebrandt, W., Thomas, H.-C., \& Ritter, H. 2004, Cox and Giuli's

Principles of Stellar Structure, ed. A. Weiss, W. Hillebrandt, H.-C. Thomas,

$\&$ H. Ritter (Cambridge, UK: Princeton Publishing Associates Ltd)

Yoon, S.-C., Langer, N., \& Scheithauer, S. 2004, A\&A, 425, 217

Zahn, J.-P. 1992, A\&A, 265, 115 
E. Glebbeek and O. R. Pols: A grid of low-mass collisions, Online Material p 1

Table 4. Results for the $Z=0.02$ collisions. The first three columns give the grid parameters, followed by the collison case (Sect. 4.3), remnant mass, mass loss parameter (see Eq. (3)), main sequence lifetimes $\tau_{\mathrm{ms}}$ for the primary, the secondary and a star of the same mass as the remnant, the main sequence lifetime of the collision product $t_{\mathrm{ms}}$ (see Sect. 6), the central hydrogen abundances $X_{\mathrm{c}}$ after the collision and at the start of the main sequence (see Sect. 4.4) and the offset of the reddest point of the main sequence track in the HRD compared to a normal star of the same mass (Sect. 6.2). Mass and luminosity are in solar units and time is measured in Myr.

\begin{tabular}{|c|c|c|c|c|c|c|c|c|c|c|c|c|c|}
\hline$t$ & $q$ & $M$ & Case & $M_{\text {remnant }}$ & $\frac{(1+q)^{2}}{q} \phi$ & $\tau_{\mathrm{ms}, 1}$ & $\tau_{\mathrm{ms}, 2}$ & $\tau_{\mathrm{ms}}$ & $t_{\mathrm{ms}}$ & $X_{\mathrm{c}, 0}$ & $X_{\mathrm{c}, \mathrm{zms}}$ & $\Delta \log _{10} L$ & $\Delta \log _{10} T_{\text {eff }}$ \\
\hline 2800 & 0.4 & 1.5 & $\mathrm{~S}$ & 1.41 & 0.28 & 6247 & 181307 & 2413 & 2100 & 0.691 & 0.676 & 0.053 & -0.004 \\
\hline 2800 & 0.4 & 1.7 & $\mathrm{P}$ & 1.60 & 0.29 & 3337 & 134060 & 1726 & 1234 & 0.379 & 0.379 & 0.074 & -0.008 \\
\hline 2800 & 0.4 & 1.8 & $\mathrm{~S}$ & 1.69 & 0.31 & 3444 & 114628 & 1478 & 951 & 0.547 & 0.574 & 0.095 & -0.009 \\
\hline 2800 & 0.4 & 1.9 & $\mathrm{P}$ & 1.77 & 0.32 & 2751 & 97623 & 1273 & 647 & 0.063 & 0.505 & 0.104 & -0.014 \\
\hline 2800 & 0.5 & 1.5 & S & 1.41 & 0.27 & 8644 & 124021 & 2519 & 2180 & 0.689 & 0.672 & 0.028 & -0.002 \\
\hline 2800 & 0.5 & 1.6 & $\mathrm{~S}$ & 1.50 & 0.29 & 6382 & 102516 & 2103 & 1709 & 0.686 & 0.666 & 0.039 & -0.001 \\
\hline 2800 & 0.5 & 1.7 & M & 1.59 & 0.29 & 4458 & 85148 & 1746 & 1395 & 0.490 & 0.490 & 0.050 & -0.006 \\
\hline 2800 & 0.5 & 1.8 & $\mathrm{P}$ & 1.69 & 0.28 & 4206 & 70071 & 1474 & 1074 & 0.415 & 0.415 & 0.058 & -0.006 \\
\hline 2800 & 0.5 & 1.9 & $\mathrm{~S}$ & 1.78 & 0.28 & 3438 & 57523 & 1261 & 1126 & 0.677 & 0.671 & 0.028 & -0.002 \\
\hline 2800 & 0.5 & 2.0 & $\mathrm{P}$ & 1.86 & 0.31 & 3001 & 47262 & 1104 & 534 & 0.124 & 0.128 & 0.065 & -0.003 \\
\hline 2800 & 0.6 & 1.5 & $\mathrm{~S}$ & 1.40 & 0.28 & 11552 & 87227 & 2608 & 2253 & 0.685 & 0.674 & 0.016 & -0.001 \\
\hline 2800 & 0.6 & 1.6 & $\mathrm{~S}$ & 1.49 & 0.31 & 8644 & 70071 & 2101 & 1848 & 0.683 & 0.662 & 0.032 & -0.001 \\
\hline 2800 & 0.6 & 1.7 & $\mathrm{~S}$ & 1.58 & 0.30 & 6504 & 56129 & 1782 & 1475 & 0.679 & 0.645 & 0.034 & -0.005 \\
\hline 2800 & 0.6 & 1.8 & $\mathrm{P}$ & 1.68 & 0.28 & 4907 & 45016 & 1485 & 1152 & 0.513 & 0.513 & 0.041 & -0.005 \\
\hline 2800 & 0.6 & 1.9 & $\mathrm{P}$ & 1.78 & 0.27 & 3734 & 36312 & 1266 & 906 & 0.422 & 0.422 & 0.049 & -0.005 \\
\hline 2800 & 0.6 & 2.0 & $\mathrm{P}$ & 1.87 & 0.29 & 3461 & 29518 & 1099 & 711 & 0.349 & 0.348 & 0.060 & -0.005 \\
\hline 2800 & 0.6 & 2.1 & $\mathrm{P}$ & 1.94 & 0.32 & 3159 & 24457 & 984 & 3 & 0.328 & 0.328 & 0.075 & -0.004 \\
\hline 2800 & 0.6 & 2.2 & $\mathrm{P}$ & 2.04 & 0.30 & 2722 & 19931 & 859 & 431 & 0.048 & 0.048 & 0.084 & -0.008 \\
\hline 2800 & 0.8 & 1.5 & $\mathrm{~S}$ & 1.40 & 0.26 & 19036 & 47262 & 2493 & 2352 & 0.676 & 0.669 & 0.016 & -0.002 \\
\hline 2800 & 0.8 & 1.6 & S & 1.49 & 0.29 & 14553 & 36598 & 2162 & 1910 & 0.672 & 0.664 & 0.014 & -0.001 \\
\hline 2800 & 0.8 & 1.7 & S & 1.57 & 0.31 & 11182 & 28644 & 1836 & 1577 & 0.662 & 0.649 & 0.016 & -0.003 \\
\hline 2800 & 0.8 & 1.8 & M & 1.67 & 0.29 & 8644 & 22659 & 1511 & 1259 & 0.586 & 0.586 & 0.021 & -0.004 \\
\hline 2800 & 0.8 & 1.9 & $\mathrm{P}$ & 1.75 & 0.31 & 6712 & 18089 & 1315 & 1051 & 0.550 & 0.550 & 0.029 & -0.005 \\
\hline 2800 & 0.8 & 2.0 & $\mathrm{P}$ & 1.86 & 0.29 & 5225 & 14553 & 1113 & 819 & 0.509 & 0.518 & 0.030 & -0.003 \\
\hline 2800 & 0.8 & 2.1 & $\mathrm{P}$ & 1.94 & 0.31 & 4861 & 11784 & 986 & 707 & 0.456 & 0.532 & 0.051 & -0.007 \\
\hline 2800 & 0.8 & 2.2 & $\mathrm{P}$ & 2.04 & 0.29 & 3956 & 9581 & 859 & 570 & 0.391 & 0.515 & 0.057 & -0.007 \\
\hline 2800 & 0.8 & 2.3 & $\mathrm{P}$ & 2.11 & 0.33 & 3445 & 7811 & 777 & 470 & 0.322 & 0.477 & 0.071 & -0.008 \\
\hline 2800 & 0.8 & 2.4 & $\mathrm{P}$ & 2.23 & 0.29 & 3001 & 6384 & 671 & 361 & 0.223 & 0.450 & 0.080 & -0.009 \\
\hline 2800 & 0.9 & 1.5 & S & 1.39 & 0.30 & 23930 & 36719 & 2643 & 2439 & 0.668 & 0.669 & 0.009 & -0.001 \\
\hline 2800 & 0.9 & 1.6 & $\mathrm{~S}$ & 1.49 & 0.28 & 18301 & 28286 & 2135 & 1920 & 0.657 & 0.661 & 0.010 & -0.002 \\
\hline 2800 & 0.9 & 1.7 & M & 1.57 & 0.31 & 14150 & 22050 & 1818 & 1586 & 0.649 & 0.649 & 0.013 & -0.002 \\
\hline 2800 & 0.9 & 1.8 & M & 1.75 & 0.11 & 11029 & 17369 & 1324 & 1055 & 0.612 & 0.611 & 0.015 & -0.002 \\
\hline 2800 & 0.9 & 1.9 & M & 1.76 & 0.31 & 8644 & 13797 & 1312 & 1071 & 0.585 & 0.620 & 0.024 & -0.005 \\
\hline 2800 & 0.9 & 2.0 & $\mathrm{P}$ & 1.86 & 0.28 & 6801 & 11029 & 1111 & 859 & 0.554 & 0.539 & 0.026 & -0.005 \\
\hline 2800 & 1.0 & 1.5 & M & 1.39 & 0.30 & 29518 & 29518 & 2689 & 2415 & 0.668 & 0.668 & 0.013 & -0.002 \\
\hline 2800 & 1.0 & 1.6 & M & 1.49 & 0.28 & 22659 & 22659 & 2149 & 1924 & 0.652 & 0.659 & 0.008 & -0.002 \\
\hline 2800 & 1.0 & 1.8 & M & 1.67 & 0.29 & 13797 & 13797 & 1512 & 1293 & 0.623 & 0.630 & 0.020 & -0.005 \\
\hline 2800 & 1.0 & 1.9 & M & 1.75 & 0.31 & 10895 & 10895 & 1317 & 1093 & 0.605 & 0.613 & 0.028 & -0.005 \\
\hline 2800 & 1.0 & 2.0 & M & 1.86 & 0.28 & 8644 & 8646 & 1110 & 854 & 0.580 & 0.591 & 0.026 & -0.004 \\
\hline 2800 & 1.0 & 2.1 & M & 1.94 & 0.30 & 6885 & 6885 & 984 & 727 & 0.552 & 0.559 & 0.038 & -0.006 \\
\hline 2800 & 1.0 & 2.2 & M & 2.05 & 0.27 & 5493 & 5493 & 848 & 583 & 0.514 & 0.518 & 0.046 & -0.006 \\
\hline 2800 & 1.0 & 2.4 & M & 2.23 & 0.29 & 4206 & 4261 & 673 & 392 & 0.412 & 0.414 & 0.070 & -0.009 \\
\hline 3100 & 0.4 & 1.5 & $\mathrm{~S}$ & 1.41 & 0.29 & 6247 & 181307 & 2443 & 2064 & 0.682 & 0.669 & 0.058 & -0.004 \\
\hline 3100 & 0.4 & 1.6 & M & 1.51 & 0.26 & 4537 & 156179 & 1869 & 1482 & 0.457 & 0.457 & 0.073 & -0.008 \\
\hline 3100 & 0.4 & 1.7 & $\mathrm{P}$ & 1.60 & 0.30 & 3337 & 134060 & 1728 & 1143 & 0.324 & 0.324 & 0.079 & -0.008 \\
\hline 3100 & 0.4 & 1.8 & $\mathrm{P}$ & 1.68 & 0.31 & 3444 & 114628 & 1487 & 864 & 0.204 & 0.204 & 0.102 & -0.008 \\
\hline 3100 & 0.5 & 1.5 & $\mathrm{~S}$ & 1.41 & 0.28 & 8644 & 124021 & 2538 & 2149 & 0.689 & 0.666 & 0.031 & -0.003 \\
\hline 3100 & 0.5 & 1.6 & $\mathrm{~S}$ & 1.50 & 0.27 & 6382 & 102516 & 2067 & 1674 & 0.659 & 0.645 & 0.041 & -0.006 \\
\hline 3100 & 0.5 & 1.7 & $\mathrm{P}$ & 1.58 & 0.31 & 4458 & 85148 & 1771 & 1341 & 0.465 & 0.465 & 0.054 & -0.006 \\
\hline 3100 & 0.5 & 1.8 & $\mathrm{P}$ & 1.69 & 0.28 & 4206 & 70071 & 1475 & 1017 & 0.365 & 0.365 & 0.064 & -0.005 \\
\hline 3100 & 0.5 & 1.9 & S & 1.78 & 0.28 & 3438 & 57523 & 1261 & 1126 & 0.677 & 0.671 & 0.028 & -0.002 \\
\hline 3100 & 0.5 & 2.0 & $\mathrm{P}$ & 1.86 & 0.31 & 3001 & 47262 & 1109 & 513 & 0.034 & 0.471 & 0.093 & -0.012 \\
\hline 3100 & 0.6 & 1.5 & S & 1.40 & 0.29 & 11552 & 87227 & 2534 & 2295 & 0.684 & 0.677 & 0.033 & -0.001 \\
\hline 3100 & 0.6 & 1.6 & $\mathrm{~S}$ & 1.49 & 0.28 & 8644 & 70071 & 2057 & 1747 & 0.681 & 0.655 & 0.028 & -0.004 \\
\hline 3100 & 0.6 & 1.7 & M & 1.58 & 0.31 & 6504 & 56129 & 1799 & 1447 & 0.540 & 0.540 & 0.036 & -0.006 \\
\hline 3100 & 0.6 & 1.9 & $\mathrm{P}$ & 1.76 & 0.31 & 3734 & 36312 & 1301 & 885 & 0.387 & 0.387 & 0.056 & -0.007 \\
\hline
\end{tabular}


Table 4. continued.

\begin{tabular}{|c|c|c|c|c|c|c|c|c|c|c|c|c|c|}
\hline$t$ & $q$ & $M$ & Case & $M_{\text {remnant }}$ & $\frac{(1+q)^{2}}{q} \phi$ & $\tau_{\mathrm{ms}, 1}$ & $\tau_{\mathrm{ms}, 2}$ & $\tau_{\mathrm{ms}}$ & $t_{\mathrm{ms}}$ & $X_{\mathrm{c}, 0}$ & $X_{\mathrm{c}, \mathrm{zms}}$ & $\Delta \log _{10} L$ & $\Delta \log _{10} T_{\text {eff }}$ \\
\hline 3100 & 0.6 & 2.0 & $\mathrm{P}$ & 1.86 & 0.30 & 3461 & 29518 & 1108 & 673 & 0.286 & 0.286 & 0.068 & -0.004 \\
\hline 3100 & 0.6 & 2.1 & $\mathrm{P}$ & 1.96 & 0.29 & 3159 & 24457 & 965 & 534 & 0.144 & 0.144 & 0.088 & -0.011 \\
\hline 3100 & 0.8 & 1.5 & S & 1.40 & 0.27 & 19036 & 47262 & 2480 & 2340 & 0.673 & 0.666 & 0.016 & -0.002 \\
\hline 3100 & 0.8 & 1.6 & $\mathrm{~S}$ & 1.48 & 0.30 & 14553 & 36598 & 2116 & 1898 & 0.666 & 0.655 & 0.017 & -0.001 \\
\hline 3100 & 0.8 & 1.7 & M & 1.59 & 0.27 & 11182 & 28644 & 1768 & 1511 & 0.666 & 0.642 & 0.019 & -0.005 \\
\hline 3100 & 0.8 & 1.8 & M & 1.67 & 0.30 & 8644 & 22659 & 1523 & 1249 & 0.573 & 0.573 & 0.026 & -0.005 \\
\hline 3100 & 0.8 & 1.9 & $\mathrm{P}$ & 1.77 & 0.27 & 6712 & 18089 & 1275 & 982 & 0.531 & 0.531 & 0.030 & -0.005 \\
\hline 3100 & 0.8 & 2.0 & $\mathrm{P}$ & 1.86 & 0.28 & 5225 & 14553 & 1104 & 777 & 0.484 & 0.558 & 0.032 & -0.004 \\
\hline 3100 & 0.8 & 2.1 & $\mathrm{P}$ & 1.93 & 0.32 & 4861 & 11784 & 997 & 680 & 0.422 & 0.508 & 0.057 & -0.007 \\
\hline 3100 & 0.8 & 2.2 & $\mathrm{P}$ & 2.04 & 0.29 & 3956 & 9581 & 855 & 534 & 0.340 & 0.486 & 0.066 & -0.008 \\
\hline 3100 & 0.8 & 2.3 & $\mathrm{P}$ & 2.14 & 0.29 & 3445 & 7811 & 756 & 434 & 0.248 & 0.463 & 0.079 & -0.008 \\
\hline 3100 & 0.8 & 2.4 & $\mathrm{P}$ & 2.22 & 0.30 & 3001 & 6384 & 676 & 1 & 0.054 & 0.054 & 0.579 & 0.048 \\
\hline 3100 & 0.9 & 1.7 & M & 1.57 & 0.31 & 14150 & 22050 & 1829 & 1568 & 0.646 & 0.643 & 0.014 & -0.002 \\
\hline 3100 & 0.9 & 1.8 & M & 1.67 & 0.29 & 11029 & 17369 & 1515 & 1265 & 0.599 & 0.628 & 0.022 & -0.005 \\
\hline 3100 & 0.9 & 2.0 & $\mathrm{P}$ & 1.85 & 0.29 & 6801 & 11029 & 1120 & 841 & 0.548 & 0.585 & 0.036 & -0.006 \\
\hline 3100 & 1.0 & 1.5 & M & 1.38 & 0.31 & 29518 & 29518 & 2588 & 2388 & 0.664 & 0.662 & 0.015 & 0.001 \\
\hline 3100 & 1.0 & 1.6 & M & 1.49 & 0.28 & 22659 & 22659 & 2095 & 1893 & 0.649 & 0.654 & 0.011 & -0.003 \\
\hline 3100 & 1.0 & 1.7 & M & 1.57 & 0.31 & 18175 & 18175 & 1840 & 1575 & 0.633 & 0.642 & 0.014 & -0.003 \\
\hline 3100 & 1.0 & 1.8 & M & 1.67 & 0.29 & 13797 & 13797 & 1520 & 1282 & 0.615 & 0.624 & 0.024 & -0.005 \\
\hline 3100 & 1.0 & 1.9 & M & 1.77 & 0.27 & 10895 & 10895 & 1276 & 1038 & 0.593 & 0.601 & 0.031 & -0.006 \\
\hline 3100 & 1.0 & 2.0 & M & 1.85 & 0.29 & 8644 & 8646 & 1122 & 847 & 0.566 & 0.586 & 0.033 & -0.006 \\
\hline 3100 & 1.0 & 2.1 & M & 1.93 & 0.31 & 6885 & 6885 & 997 & 705 & 0.535 & 0.541 & 0.042 & -0.007 \\
\hline 3100 & 1.0 & 2.2 & M & 2.04 & 0.29 & 5493 & 5493 & 862 & 566 & 0.491 & 0.488 & 0.053 & -0.007 \\
\hline 3100 & 1.0 & 2.4 & M & 2.21 & 0.31 & 4206 & 4261 & 682 & 363 & 0.378 & 0.379 & 0.078 & -0.011 \\
\hline 3400 & 0.4 & 1.2 & S & 1.13 & 0.29 & 16991 & 314052 & 5263 & 4416 & 0.698 & 0.690 & 0.023 & 0.000 \\
\hline 3400 & 0.4 & 1.3 & $\mathrm{~S}$ & 1.23 & 0.28 & 12092 & 243954 & 3952 & 3439 & 0.691 & 0.683 & 0.028 & -0.000 \\
\hline 3400 & 0.4 & 1.4 & S & 1.32 & 0.27 & 8644 & 210585 & 3089 & 2568 & 0.690 & 0.676 & 0.042 & -0.002 \\
\hline 3400 & 0.4 & 1.5 & $\mathrm{~S}$ & 1.42 & 0.27 & 6247 & 181307 & 2495 & 1968 & 0.689 & 0.657 & 0.055 & -0.004 \\
\hline 3400 & 0.4 & 1.6 & $\mathrm{P}$ & 1.50 & 0.30 & 4537 & 156179 & 2087 & 1486 & 0.411 & 0.411 & 0.070 & -0.006 \\
\hline 3400 & 0.4 & 1.7 & $\mathrm{P}$ & 1.60 & 0.28 & 3337 & 134060 & 1710 & 1038 & 0.262 & 0.262 & 0.080 & -0.007 \\
\hline 3400 & 0.4 & 1.8 & $\mathrm{P}$ & 1.69 & 0.29 & 3444 & 114628 & 1467 & 624 & 0.106 & 0.110 & 0.078 & 0.002 \\
\hline 3400 & 0.5 & 1.5 & $\mathrm{~S}$ & 1.40 & 0.29 & 8644 & 124021 & 2555 & 2128 & 0.681 & 0.661 & 0.034 & -0.003 \\
\hline 3400 & 0.5 & 1.6 & M & 1.50 & 0.28 & 6382 & 102516 & 2088 & 1682 & 0.502 & 0.639 & 0.048 & -0.005 \\
\hline 3400 & 0.5 & 1.7 & $\mathrm{P}$ & 1.59 & 0.30 & 4458 & 85148 & 1762 & 1268 & 0.430 & 0.430 & 0.058 & -0.007 \\
\hline 3400 & 0.5 & 1.8 & $\mathrm{P}$ & 1.68 & 0.30 & 4206 & 70071 & 1486 & 927 & 0.309 & 0.310 & 0.069 & -0.006 \\
\hline 3400 & 0.5 & 1.9 & S & 1.78 & 0.28 & 3438 & 57523 & 1262 & 1129 & 0.676 & 0.671 & 0.028 & -0.002 \\
\hline 3400 & 0.6 & 1.2 & S & 1.12 & 0.27 & 29518 & 162623 & 5079 & 4986 & 0.689 & 0.688 & 0.022 & -0.001 \\
\hline 3400 & 0.6 & 1.3 & S & 1.22 & 0.27 & 21253 & 133093 & 4005 & 3692 & 0.688 & 0.681 & 0.018 & -0.001 \\
\hline 3400 & 0.6 & 1.4 & S & 1.31 & 0.26 & 15571 & 108190 & 3152 & 2812 & 0.686 & 0.674 & 0.018 & -0.001 \\
\hline 3400 & 0.6 & 1.5 & S & 1.40 & 0.30 & 11552 & 87227 & 2539 & 2257 & 0.684 & 0.664 & 0.029 & -0.002 \\
\hline 3400 & 0.6 & 1.6 & S & 1.49 & 0.29 & 8644 & 70071 & 2131 & 1721 & 0.679 & 0.648 & 0.030 & -0.004 \\
\hline 3400 & 0.6 & 1.7 & $\mathrm{P}$ & 1.59 & 0.27 & 6504 & 56129 & 1746 & 1367 & 0.509 & 0.509 & 0.040 & -0.006 \\
\hline 3400 & 0.6 & 1.8 & $\mathrm{P}$ & 1.68 & 0.28 & 4907 & 45016 & 1487 & 1065 & 0.440 & 0.440 & 0.049 & -0.006 \\
\hline 3400 & 0.6 & 1.9 & $\mathrm{P}$ & 1.76 & 0.31 & 3734 & 36312 & 1301 & 830 & 0.335 & 0.335 & 0.060 & -0.007 \\
\hline 3400 & 0.6 & 2.0 & $\mathrm{P}$ & 1.86 & 0.30 & 3461 & 29518 & 1115 & 606 & 0.206 & 0.203 & 0.071 & -0.004 \\
\hline 3400 & 0.6 & 2.1 & $\mathrm{P}$ & 1.93 & 0.34 & 3159 & 24457 & 1000 & 12 & 0.037 & 0.037 & 0.095 & -0.009 \\
\hline 3400 & 0.8 & 1.2 & S & 1.12 & 0.26 & 47280 & 102516 & 4972 & 3149 & 0.686 & 0.684 & 0.008 & 0.009 \\
\hline 3400 & 0.8 & 1.3 & S & 1.21 & 0.29 & 34390 & 79923 & 4135 & 16 & 0.682 & 0.680 & 0.000 & 0.001 \\
\hline 3400 & 0.8 & 1.4 & $\mathrm{~S}$ & 1.30 & 0.29 & 25450 & 61494 & 3244 & 2995 & 0.679 & 0.673 & 0.011 & -0.001 \\
\hline 3400 & 0.8 & 1.5 & $\mathrm{~S}$ & 1.40 & 0.27 & 19036 & 47262 & 2492 & 2323 & 0.673 & 0.661 & 0.017 & -0.002 \\
\hline 3400 & 0.8 & 1.6 & $\mathrm{~S}$ & 1.48 & 0.30 & 14553 & 36598 & 2126 & 1886 & 0.667 & 0.651 & 0.018 & -0.002 \\
\hline 3400 & 0.8 & 1.7 & M & 1.58 & 0.28 & 11182 & 28644 & 1780 & 1494 & 0.596 & 0.596 & 0.022 & -0.005 \\
\hline 3400 & 0.8 & 1.8 & $\mathrm{P}$ & 1.68 & 0.28 & 8644 & 22659 & 1499 & 1192 & 0.553 & 0.553 & 0.028 & -0.005 \\
\hline 3400 & 0.8 & 1.9 & $\mathrm{P}$ & 1.76 & 0.31 & 6712 & 18089 & 1311 & 968 & 0.513 & 0.510 & 0.031 & -0.005 \\
\hline 3400 & 0.8 & 2.0 & $\mathrm{P}$ & 1.85 & 0.29 & 5225 & 14553 & 1120 & 782 & 0.455 & 0.559 & 0.046 & -0.007 \\
\hline 3400 & 0.8 & 2.1 & $\mathrm{P}$ & 1.95 & 0.29 & 4861 & 11784 & 970 & 628 & 0.378 & 0.493 & 0.060 & -0.009 \\
\hline 3400 & 0.8 & 2.2 & $\mathrm{P}$ & 2.03 & 0.32 & 3956 & 9581 & 877 & 503 & 0.297 & 0.452 & 0.075 & -0.009 \\
\hline 3400 & 0.8 & 2.3 & $\mathrm{P}$ & 2.13 & 0.29 & 3445 & 7811 & 759 & 385 & 0.185 & 0.391 & 0.085 & -0.010 \\
\hline 3400 & 0.8 & 2.4 & $\mathrm{P}$ & 2.18 & 0.37 & 3001 & 6384 & 709 & 287 & 0.201 & 0.201 & 0.109 & -0.015 \\
\hline 3400 & 0.9 & 1.7 & M & 1.59 & 0.27 & 14150 & 22050 & 1764 & 1531 & 0.609 & 0.621 & 0.022 & -0.004 \\
\hline 3400 & 0.9 & 1.8 & M & 1.67 & 0.30 & 11029 & 17369 & 1526 & 1245 & 0.586 & 0.584 & 0.023 & -0.006 \\
\hline
\end{tabular}


Table 4. continued.

\begin{tabular}{|c|c|c|c|c|c|c|c|c|c|c|c|c|c|}
\hline$t$ & $q$ & $M$ & Case & $M_{\text {remnant }}$ & $\frac{(1+q)^{2}}{q} \phi$ & $\tau_{\mathrm{ms}, 1}$ & $\tau_{\mathrm{ms}, 2}$ & $\tau_{\mathrm{ms}}$ & $t_{\mathrm{ms}}$ & $X_{\mathrm{c}, 0}$ & $X_{\mathrm{c}, \mathrm{zms}}$ & $\Delta \log _{10} L$ & $\Delta \log _{10} T_{\text {eff }}$ \\
\hline 3400 & 0.9 & 1.9 & $\mathrm{P}$ & 1.77 & 0.28 & 8644 & 13797 & 1280 & 1002 & 0.559 & 0.597 & 0.032 & -0.006 \\
\hline 3400 & 0.9 & 2.0 & $\mathrm{P}$ & 1.86 & 0.28 & 6801 & 11029 & 1110 & 801 & 0.510 & 0.569 & 0.037 & -0.007 \\
\hline 3400 & 1.0 & 1.2 & M & 1.12 & 0.28 & 70143 & 70071 & 5094 & 4783 & 0.681 & 0.682 & 0.001 & -0.000 \\
\hline 3400 & 1.0 & 1.3 & M & 1.20 & 0.31 & 52156 & 52156 & 4217 & 3971 & 0.677 & 0.678 & 0.006 & -0.000 \\
\hline 3400 & 1.0 & 1.4 & M & 1.30 & 0.28 & 38969 & 38969 & 3243 & 2928 & 0.668 & 0.670 & 0.007 & 0.001 \\
\hline 3400 & 1.0 & 1.5 & M & 1.38 & 0.31 & 29518 & 29518 & 2691 & 2384 & 0.659 & 0.659 & 0.013 & -0.001 \\
\hline 3400 & 1.0 & 1.6 & M & 1.48 & 0.29 & 22659 & 22659 & 2166 & 1894 & 0.646 & 0.649 & 0.012 & -0.002 \\
\hline 3400 & 1.0 & 1.7 & M & 1.59 & 0.27 & 18175 & 18175 & 1753 & 1510 & 0.624 & 0.635 & 0.019 & -0.005 \\
\hline 3400 & 1.0 & 1.8 & M & 1.67 & 0.30 & 13797 & 13797 & 1528 & 1227 & 0.620 & 0.605 & 0.013 & -0.006 \\
\hline 3400 & 1.0 & 2.0 & M & 1.85 & 0.30 & 8644 & 8646 & 1132 & 818 & 0.552 & 0.574 & 0.033 & -0.006 \\
\hline 3400 & 1.0 & 2.1 & M & 1.95 & 0.28 & 6885 & 6885 & 970 & 662 & 0.512 & 0.519 & 0.048 & -0.007 \\
\hline 3400 & 1.0 & 2.2 & M & 2.03 & 0.32 & 5493 & 5493 & 876 & 541 & 0.467 & 0.470 & 0.059 & -0.008 \\
\hline 3400 & 1.0 & 2.4 & M & 2.22 & 0.30 & 4206 & 4261 & 676 & 328 & 0.325 & 0.326 & 0.085 & -0.013 \\
\hline 3700 & 0.4 & 1.2 & $\mathrm{~S}$ & 1.14 & 0.25 & 16991 & 314052 & 4881 & 4436 & 0.698 & 0.687 & 0.039 & -0.000 \\
\hline 3700 & 0.4 & 1.3 & S & 1.22 & 0.29 & 12092 & 243954 & 3962 & 3405 & 0.691 & 0.682 & 0.034 & -0.000 \\
\hline 3700 & 0.4 & 1.4 & S & 1.32 & 0.29 & 8644 & 210585 & 3113 & 2540 & 0.690 & 0.674 & 0.046 & -0.002 \\
\hline 3700 & 0.4 & 1.5 & S & 1.41 & 0.28 & 6247 & 181307 & 2526 & 1932 & 0.667 & 0.648 & 0.061 & -0.004 \\
\hline 3700 & 0.4 & 1.8 & $\mathrm{P}$ & 1.68 & 0.34 & 3444 & 114628 & 1501 & 667 & 0.032 & 0.467 & 0.114 & -0.017 \\
\hline 3700 & 0.5 & 1.5 & $\mathrm{~S}$ & 1.41 & 0.26 & 8644 & 124021 & 2507 & 2054 & 0.678 & 0.656 & 0.038 & -0.004 \\
\hline 3700 & 0.5 & 1.6 & $\mathrm{P}$ & 1.49 & 0.30 & 6382 & 102516 & 2111 & 1610 & 0.479 & 0.479 & 0.050 & -0.006 \\
\hline 3700 & 0.5 & 1.7 & $\mathrm{P}$ & 1.59 & 0.29 & 4458 & 85148 & 1747 & 1196 & 0.391 & 0.391 & 0.063 & -0.007 \\
\hline 3700 & 0.5 & 1.8 & $\mathrm{P}$ & 1.68 & 0.31 & 4206 & 70071 & 1494 & 850 & 0.251 & 0.251 & 0.070 & -0.006 \\
\hline 3700 & 0.5 & 1.9 & S & 1.78 & 0.28 & 3438 & 57523 & 1263 & 1120 & 0.672 & 0.669 & 0.029 & -0.002 \\
\hline 3700 & 0.6 & 1.2 & S & 1.12 & 0.27 & 29518 & 162623 & 4958 & 2885 & 0.689 & 0.686 & 0.008 & 0.001 \\
\hline 3700 & 0.6 & 1.3 & S & 1.22 & 0.27 & 21253 & 133093 & 4010 & 3642 & 0.688 & 0.679 & 0.018 & -0.000 \\
\hline 3700 & 0.6 & 1.4 & $\mathrm{~S}$ & 1.31 & 0.27 & 15571 & 108190 & 3181 & 2796 & 0.686 & 0.672 & 0.018 & -0.001 \\
\hline 3700 & 0.6 & 1.5 & $\mathrm{~S}$ & 1.41 & 0.27 & 11552 & 87227 & 2479 & 2176 & 0.680 & 0.660 & 0.033 & -0.002 \\
\hline 3700 & 0.6 & 1.6 & M & 1.49 & 0.30 & 8644 & 70071 & 2094 & 1699 & 0.677 & 0.645 & 0.035 & -0.004 \\
\hline 3700 & 0.6 & 1.7 & $\mathrm{P}$ & 1.59 & 0.28 & 6504 & 56129 & 1765 & 1335 & 0.501 & 0.501 & 0.042 & -0.006 \\
\hline 3700 & 0.6 & 1.8 & $\mathrm{P}$ & 1.68 & 0.28 & 4907 & 45016 & 1480 & 1010 & 0.403 & 0.403 & 0.052 & -0.007 \\
\hline 3700 & 0.6 & 1.9 & $\mathrm{P}$ & 1.77 & 0.29 & 3734 & 36312 & 1275 & 753 & 0.283 & 0.283 & 0.060 & -0.006 \\
\hline 3700 & 0.6 & 2.0 & $\mathrm{P}$ & 1.85 & 0.33 & 3461 & 29518 & 1133 & 556 & 0.120 & 0.134 & 0.094 & -0.012 \\
\hline 3700 & 0.8 & 1.2 & S & 1.12 & 0.26 & 47280 & 102516 & 4972 & 3149 & 0.686 & 0.684 & 0.001 & 0.000 \\
\hline 3700 & 0.8 & 1.3 & S & 1.21 & 0.29 & 34390 & 79923 & 4160 & 3844 & 0.682 & 0.678 & 0.003 & -0.000 \\
\hline 3700 & 0.8 & 1.4 & $\mathrm{~S}$ & 1.30 & 0.29 & 25450 & 61494 & 3261 & 2972 & 0.676 & 0.669 & 0.012 & -0.001 \\
\hline 3700 & 0.8 & 1.5 & $\mathrm{~S}$ & 1.40 & 0.28 & 19036 & 47262 & 2624 & 2303 & 0.658 & 0.657 & 0.009 & -0.002 \\
\hline 3700 & 0.8 & 1.6 & M & 1.48 & 0.31 & 14553 & 36598 & 2137 & 1862 & 0.659 & 0.644 & 0.018 & -0.002 \\
\hline 3700 & 0.8 & 1.7 & M & 1.58 & 0.29 & 11182 & 28644 & 1790 & 1466 & 0.589 & 0.589 & 0.022 & -0.005 \\
\hline 3700 & 0.8 & 1.8 & $\mathrm{P}$ & 1.67 & 0.29 & 8644 & 22659 & 1512 & 1172 & 0.541 & 0.541 & 0.030 & -0.006 \\
\hline 3700 & 0.8 & 1.9 & $\mathrm{P}$ & 1.75 & 0.32 & 6712 & 18089 & 1325 & 931 & 0.492 & 0.526 & 0.032 & -0.004 \\
\hline 3700 & 0.8 & 2.0 & $\mathrm{P}$ & 1.84 & 0.32 & 5225 & 14553 & 1141 & 761 & 0.428 & 0.545 & 0.050 & -0.008 \\
\hline 3700 & 0.8 & 2.1 & $\mathrm{P}$ & 1.94 & 0.30 & 4861 & 11784 & 985 & 619 & 0.345 & 0.463 & 0.071 & -0.008 \\
\hline 3700 & 0.8 & 2.2 & $\mathrm{P}$ & 2.03 & 0.31 & 3956 & 9581 & 869 & 481 & 0.233 & 0.425 & 0.081 & -0.010 \\
\hline 3700 & 0.8 & 2.3 & $\mathrm{P}$ & 2.12 & 0.32 & 3445 & 7811 & 772 & 1 & 0.050 & 0.050 & 0.153 & 0.060 \\
\hline 3700 & 0.9 & 1.5 & $\mathrm{~S}$ & 1.40 & 0.26 & 23930 & 36719 & 2528 & 2296 & 0.654 & 0.657 & 0.013 & -0.003 \\
\hline 3700 & 0.9 & 1.6 & M & 1.48 & 0.29 & 18301 & 28286 & 2160 & 1871 & 0.650 & 0.647 & 0.014 & -0.003 \\
\hline 3700 & 0.9 & 1.7 & M & 1.57 & 0.30 & 14150 & 22050 & 1808 & 1497 & 0.599 & 0.599 & 0.017 & -0.004 \\
\hline 3700 & 0.9 & 1.8 & M & 1.67 & 0.28 & 11029 & 17369 & 1503 & 1204 & 0.572 & 0.572 & 0.026 & -0.006 \\
\hline 3700 & 0.9 & 1.9 & $\mathrm{P}$ & 1.75 & 0.31 & 8644 & 13797 & 1319 & 958 & 0.541 & 0.582 & 0.026 & -0.004 \\
\hline 3700 & 0.9 & 2.0 & $\mathrm{P}$ & 1.85 & 0.29 & 6801 & 11029 & 1121 & 777 & 0.492 & 0.558 & 0.040 & -0.006 \\
\hline 3700 & 1.0 & 1.2 & M & 1.12 & 0.28 & 70143 & 70071 & 5094 & 4783 & 0.681 & 0.682 & 0.001 & -0.000 \\
\hline 3700 & 1.0 & 1.3 & M & 1.20 & 0.31 & 52156 & 52156 & 4228 & 4030 & 0.675 & 0.675 & 0.001 & 0.000 \\
\hline 3700 & 1.0 & 1.4 & M & 1.30 & 0.28 & 38969 & 38969 & 3236 & 2988 & 0.665 & 0.667 & 0.009 & -0.001 \\
\hline 3700 & 1.0 & 1.5 & M & 1.40 & 0.26 & 29518 & 29518 & 2598 & 2268 & 0.650 & 0.651 & 0.010 & -0.000 \\
\hline 3700 & 1.0 & 1.6 & M & 1.48 & 0.29 & 22659 & 22659 & 2178 & 1878 & 0.636 & 0.645 & 0.013 & -0.002 \\
\hline 3700 & 1.0 & 1.7 & M & 1.58 & 0.28 & 18175 & 18175 & 1780 & 1490 & 0.618 & 0.625 & 0.018 & -0.005 \\
\hline 3700 & 1.0 & 1.8 & M & 1.66 & 0.31 & 13797 & 13797 & 1542 & 1196 & 0.597 & 0.596 & 0.013 & -0.005 \\
\hline 3700 & 1.0 & 1.9 & M & 1.76 & 0.29 & 10895 & 10895 & 1296 & 997 & 0.572 & 0.582 & 0.036 & -0.007 \\
\hline 3700 & 1.0 & 2.0 & M & 1.84 & 0.32 & 8644 & 8646 & 1136 & 783 & 0.537 & 0.554 & 0.035 & -0.005 \\
\hline 3700 & 1.0 & 2.1 & M & 1.94 & 0.30 & 6885 & 6885 & 983 & 641 & 0.493 & 0.492 & 0.053 & -0.008 \\
\hline 3700 & 1.0 & 2.2 & M & 2.04 & 0.29 & 5493 & 5493 & 861 & 411 & 0.436 & 0.436 & 0.001 & 0.000 \\
\hline
\end{tabular}


E. Glebbeek and O. R. Pols: A grid of low-mass collisions, Online Material p 4

Table 4. continued.

\begin{tabular}{|c|c|c|c|c|c|c|c|c|c|c|c|c|c|}
\hline$t$ & $q$ & $M$ & Case & $M_{\text {remnant }}$ & $\frac{(1+q)^{2}}{q} \phi$ & $\tau_{\mathrm{ms}, 1}$ & $\tau_{\mathrm{ms}, 2}$ & $\tau_{\mathrm{ms}}$ & $t_{\mathrm{ms}}$ & $X_{\mathrm{c}, 0}$ & $X_{\mathrm{c}, \mathrm{zms}}$ & $\Delta \log _{10} L$ & $\Delta \log _{10} T_{\mathrm{eff}}$ \\
\hline 3700 & 1.0 & 2.4 & $\mathrm{M}$ & 2.20 & 0.34 & 4206 & 4261 & 695 & 305 & 0.281 & 0.281 & 0.098 & -0.015 \\
\hline 4200 & 0.4 & 1.2 & $\mathrm{~S}$ & 1.14 & 0.26 & 16991 & 314052 & 5119 & 4442 & 0.698 & 0.684 & 0.031 & 0.000 \\
\hline 4200 & 0.4 & 1.3 & $\mathrm{~S}$ & 1.23 & 0.26 & 12092 & 243954 & 3890 & 3272 & 0.689 & 0.676 & 0.037 & -0.001 \\
\hline 4200 & 0.4 & 1.4 & $\mathrm{~S}$ & 1.33 & 0.26 & 8644 & 210585 & 3052 & 2379 & 0.688 & 0.666 & 0.058 & -0.000 \\
\hline 4200 & 0.6 & 1.2 & $\mathrm{~S}$ & 1.12 & 0.29 & 29518 & 162623 & 5056 & 4728 & 0.688 & 0.684 & 0.028 & 0.000 \\
\hline 4200 & 0.6 & 1.3 & $\mathrm{~S}$ & 1.22 & 0.28 & 21253 & 133093 & 4050 & 3623 & 0.685 & 0.676 & 0.018 & -0.001 \\
\hline 4200 & 0.6 & 1.4 & $\mathrm{~S}$ & 1.31 & 0.28 & 15571 & 108190 & 3191 & 2755 & 0.683 & 0.666 & 0.022 & -0.002 \\
\hline 4200 & 0.8 & 1.2 & $\mathrm{~S}$ & 1.12 & 0.27 & 47280 & 102516 & 5003 & 4701 & 0.683 & 0.681 & 0.014 & 0.000 \\
\hline 4200 & 0.8 & 1.3 & $\mathrm{~S}$ & 1.20 & 0.30 & 34390 & 79923 & 4157 & 3820 & 0.679 & 0.675 & 0.005 & -0.000 \\
\hline 4200 & 0.8 & 1.4 & $\mathrm{~S}$ & 1.30 & 0.30 & 25450 & 61494 & 3273 & 2948 & 0.673 & 0.665 & 0.013 & -0.001 \\
\hline 4200 & 1.0 & 1.2 & M & 1.12 & 0.28 & 70143 & 70071 & 5117 & 4757 & 0.678 & 0.679 & 0.001 & 0.000 \\
\hline 4200 & 1.0 & 1.3 & M & 1.22 & 0.26 & 52156 & 52156 & 4049 & 270 & 0.670 & 0.671 & 0.004 & 0.000 \\
\hline 4200 & 1.0 & 1.4 & M & 1.30 & 0.29 & 38969 & 38969 & 3256 & 2953 & 0.659 & 0.661 & 0.010 & -0.001 \\
\hline 4200 & 1.0 & 2.2 & $\mathrm{M}$ & 2.04 & 0.30 & 5493 & 5493 & 864 & 454 & 0.386 & 0.386 & 0.083 & -0.011 \\
\hline 4700 & 0.4 & 1.2 & $\mathrm{~S}$ & 1.13 & 0.27 & 16991 & 314052 & 4934 & 4420 & 0.698 & 0.682 & 0.062 & -0.000 \\
\hline 4700 & 0.4 & 1.3 & $\mathrm{~S}$ & 1.23 & 0.28 & 12092 & 243954 & 3930 & 3213 & 0.687 & 0.671 & 0.042 & -0.001 \\
\hline 4700 & 0.4 & 1.4 & $\mathrm{~S}$ & 1.32 & 0.28 & 8644 & 210585 & 3103 & 2310 & 0.686 & 0.669 & 0.058 & -0.003 \\
\hline 4700 & 0.6 & 1.2 & $\mathrm{~S}$ & 1.13 & 0.25 & 29518 & 162623 & 5176 & 4571 & 0.684 & 0.681 & 0.006 & 0.001 \\
\hline 4700 & 0.6 & 1.3 & $\mathrm{~S}$ & 1.21 & 0.28 & 21253 & 133093 & 4072 & 12 & 0.685 & 0.673 & 0.007 & 0.000 \\
\hline 4700 & 0.6 & 1.4 & $\mathrm{~S}$ & 1.31 & 0.29 & 15571 & 108190 & 3215 & 2720 & 0.680 & 0.660 & 0.025 & -0.002 \\
\hline 4700 & 0.8 & 1.2 & $\mathrm{~S}$ & 1.12 & 0.27 & 47280 & 102516 & 5023 & 4810 & 0.680 & 0.678 & 0.021 & 0.000 \\
\hline 4700 & 0.8 & 1.3 & $\mathrm{~S}$ & 1.20 & 0.30 & 34390 & 79923 & 486 & 14 & 0.676 & 0.671 & 0.000 & 0.000 \\
\hline 4700 & 0.8 & 1.4 & $\mathrm{~S}$ & 1.29 & 0.31 & 25450 & 61494 & 3299 & 3009 & 0.670 & 0.659 & 0.035 & -0.006 \\
\hline 4700 & 1.0 & 1.2 & $\mathrm{M}$ & 1.11 & 0.28 & 70143 & 70071 & 5140 & 4725 & 0.675 & 0.676 & 0.000 & -0.000 \\
\hline 4700 & 1.0 & 1.3 & M & 1.21 & 0.27 & 52156 & 52156 & 4059 & 3848 & 0.667 & 0.667 & 0.006 & -0.000 \\
\hline 4700 & 1.0 & 1.4 & M & 1.30 & 0.30 & 38969 & 38969 & 3275 & 2922 & 0.652 & 0.655 & 0.011 & -0.001 \\
\hline 4700 & 1.0 & 2.2 & $\mathrm{M}$ & 2.03 & 0.32 & 5493 & 5493 & 877 & 431 & 0.329 & 0.329 & 0.100 & -0.015 \\
\hline 5200 & 0.4 & 1.2 & $\mathrm{~S}$ & 1.13 & 0.29 & 16991 & 314052 & 5253 & 4375 & 0.698 & 0.680 & 0.044 & 0.001 \\
\hline 5200 & 0.4 & 1.3 & $\mathrm{~S}$ & 1.22 & 0.29 & 12092 & 243954 & 3955 & 3166 & 0.687 & 0.669 & 0.050 & -0.001 \\
\hline 5200 & 0.4 & 1.4 & M & 1.33 & 0.26 & 8644 & 210585 & 3061 & 2251 & 0.447 & 0.421 & 0.067 & -0.004 \\
\hline 5200 & 0.6 & 1.2 & $\mathrm{~S}$ & 1.13 & 0.25 & 29518 & 162623 & 5250 & 4687 & 0.684 & 0.679 & 0.020 & 0.000 \\
\hline 5200 & 0.6 & 1.3 & $\mathrm{~S}$ & 1.22 & 0.26 & 21253 & 133093 & 3970 & 3471 & 0.682 & 0.668 & 0.020 & -0.001 \\
\hline 5200 & 0.6 & 1.4 & $\mathrm{~S}$ & 1.31 & 0.26 & 15571 & 108190 & 3153 & 2628 & 0.679 & 0.653 & 0.029 & -0.002 \\
\hline 5200 & 0.8 & 1.2 & $\mathrm{~S}$ & 1.12 & 0.27 & 47280 & 102516 & 5048 & 2917 & 0.680 & 0.676 & 0.000 & 0.000 \\
\hline 5200 & 0.8 & 1.3 & $\mathrm{~S}$ & 1.22 & 0.26 & 34390 & 79923 & 75 & 3614 & 0.672 & 0.666 & 0.277 & -0.006 \\
\hline 5200 & 0.8 & 1.4 & $\mathrm{~S}$ & 1.29 & 0.31 & 25450 & 61494 & 3311 & 2888 & 0.667 & 0.653 & 0.016 & -0.002 \\
\hline 5200 & 1.0 & 1.2 & M & 1.11 & 0.29 & 70143 & 70071 & 5162 & 4703 & 0.672 & 0.673 & 0.001 & -0.000 \\
\hline 5200 & 1.0 & 1.3 & $\mathrm{M}$ & 1.21 & 0.27 & 52156 & 52156 & 4073 & 3689 & 0.661 & 0.662 & 0.009 & -0.001 \\
\hline 5200 & 1.0 & 1.4 & M & 1.30 & 0.30 & 38969 & 38969 & 3286 & 2910 & 0.649 & 0.652 & 0.012 & -0.001 \\
\hline 5200 & 1.0 & 2.2 & M & 2.02 & 0.32 & 5493 & 5493 & 880 & 379 & 0.263 & 0.263 & 0.125 & -0.018 \\
\hline 5700 & 0.4 & 1.2 & $\mathrm{~S}$ & 1.13 & 0.30 & 16991 & 314052 & 4884 & 3914 & 0.698 & 0.677 & 0.009 & -0.002 \\
\hline 5700 & 0.4 & 1.3 & $\mathrm{~S}$ & 1.23 & 0.26 & 12092 & 243954 & 3897 & 3022 & 0.684 & 0.661 & 0.053 & -0.001 \\
\hline 5700 & 0.6 & 1.2 & $\mathrm{~S}$ & 1.13 & 0.26 & 29518 & 162623 & 4626 & 4641 & 0.681 & 0.675 & 0.081 & -0.001 \\
\hline 5700 & 0.6 & 1.3 & $\mathrm{~S}$ & 1.22 & 0.26 & 21253 & 133093 & 3551 & 3463 & 0.679 & 0.664 & 0.125 & 0.005 \\
\hline 5700 & 0.6 & 1.4 & $\mathrm{~S}$ & 1.31 & 0.27 & 15571 & 108190 & 3174 & 2592 & 0.676 & 0.647 & 0.032 & -0.003 \\
\hline 5700 & 0.8 & 1.2 & $\mathrm{~S}$ & 1.12 & 0.28 & 47280 & 102516 & 5068 & 4926 & 0.677 & 0.673 & 0.041 & -0.001 \\
\hline 5700 & 0.8 & 1.3 & $\mathrm{~S}$ & 1.22 & 0.26 & 34390 & 79923 & 4040 & 13 & 0.669 & 0.662 & 0.004 & 0.000 \\
\hline 5700 & 0.8 & 1.4 & $\mathrm{~S}$ & 1.31 & 0.27 & 25450 & 61494 & 3197 & 2742 & 0.662 & 0.648 & 0.014 & -0.002 \\
\hline 5700 & 1.0 & 1.2 & $\mathrm{M}$ & 1.11 & 0.29 & 70143 & 70071 & 5183 & 4664 & 0.669 & 0.671 & 0.000 & -0.000 \\
\hline 5700 & 1.0 & 1.3 & M & 1.21 & 0.27 & 52156 & 52156 & 4127 & 3669 & 0.658 & 0.659 & 0.004 & -0.000 \\
\hline 5700 & 1.0 & 1.4 & M & 1.29 & 0.31 & 38969 & 38969 & 3304 & 2876 & 0.643 & 0.646 & 0.014 & -0.001 \\
\hline
\end{tabular}


Table 5. As Table 4 for the $Z=0.001$ models.

\begin{tabular}{|c|c|c|c|c|c|c|c|c|c|c|c|c|c|}
\hline$t$ & $q$ & $M$ & Case & $M_{\text {remnant }}$ & $\frac{(1+q)^{2}}{q} \phi$ & $\tau_{\mathrm{ms}, 1}$ & $\tau_{\mathrm{ms}, 2}$ & $\tau_{\mathrm{ms}}$ & $t_{\mathrm{ms}}$ & $X_{\mathrm{c}, 0}$ & $X_{\mathrm{c}, \mathrm{zms}}$ & $\Delta \log _{10} L$ & $\Delta \log _{10} T_{\text {eff }}$ \\
\hline 8000 & 0.4 & 0.8 & $\mathrm{~S}$ & 0.76 & 0.25 & 43810 & 695587 & 14678 & 13000 & 0.753 & 0.751 & 0.018 & 0.001 \\
\hline 8000 & 0.4 & 0.9 & $\mathrm{~S}$ & 0.85 & 0.25 & 27922 & 544208 & 9331 & 7683 & 0.752 & 0.719 & 0.032 & 0.002 \\
\hline 8000 & 0.4 & 1.0 & $\mathrm{~S}$ & 0.95 & 0.25 & 18612 & 418946 & 6214 & 4645 & 0.752 & 0.701 & 0.062 & 0.006 \\
\hline 8000 & 0.4 & 1.1 & $\mathrm{P}$ & 1.04 & 0.27 & 12867 & 313659 & 4353 & 2459 & 0.408 & 0.403 & 0.066 & 0.010 \\
\hline 8000 & 0.4 & 1.2 & $\mathrm{P}$ & 1.13 & 0.29 & 9181 & 221923 & 3150 & 3 & 0.210 & 0.210 & 0.000 & 0.089 \\
\hline 8000 & 0.6 & 0.8 & $\mathrm{~S}$ & 0.76 & 0.24 & 72663 & 364938 & 14963 & 13905 & 0.752 & 0.752 & 0.015 & 0.001 \\
\hline 8000 & 0.6 & 0.9 & $\mathrm{~S}$ & 0.85 & 0.24 & 46528 & 236659 & 9496 & 8484 & 0.751 & 0.746 & 0.016 & 0.001 \\
\hline 8000 & 0.6 & 1.0 & $\mathrm{~S}$ & 0.93 & 0.28 & 31103 & 178559 & 6579 & 5470 & 0.730 & 0.722 & 0.019 & 0.001 \\
\hline 8000 & 0.6 & 1.1 & M & 1.03 & 0.27 & 21571 & 138218 & 4513 & 2827 & 0.573 & 0.541 & 0.030 & 0.001 \\
\hline 8000 & 0.6 & 1.2 & $\mathrm{P}$ & 1.12 & 0.28 & 15409 & 105374 & 3241 & 1986 & 0.485 & 0.485 & 0.027 & 0.004 \\
\hline 8000 & 0.6 & 1.3 & $\mathrm{P}$ & 1.22 & 0.28 & 11298 & 79734 & 2354 & 935 & 0.348 & 0.350 & 0.028 & 0.006 \\
\hline 8000 & 0.8 & 0.8 & $\mathrm{~S}$ & 0.75 & 0.25 & 109778 & 202645 & 15353 & 14290 & 0.735 & 0.733 & 0.004 & 0.000 \\
\hline 8000 & 0.8 & 0.9 & $\mathrm{~S}$ & 0.85 & 0.24 & 72663 & 150804 & 9633 & 8725 & 0.727 & 0.727 & 0.004 & 0.000 \\
\hline 8000 & 0.8 & 1.0 & $\mathrm{~S}$ & 0.94 & 0.25 & 48790 & 109778 & 6487 & 5614 & 0.720 & 0.713 & 0.006 & 0.000 \\
\hline 8000 & 0.8 & 1.2 & $\mathrm{P}$ & 1.12 & 0.26 & 24284 & 57012 & 3215 & 2305 & 0.592 & 0.592 & 0.011 & 0.001 \\
\hline 8000 & 0.8 & 1.3 & $\mathrm{P}$ & 1.21 & 0.30 & 17831 & 41997 & 2441 & 1493 & 0.526 & 0.527 & 0.025 & 0.002 \\
\hline 8000 & 1.0 & 0.8 & M & 0.75 & 0.26 & 150804 & 150804 & 15550 & 14471 & 0.729 & 0.729 & 0.003 & 0.000 \\
\hline 8000 & 1.0 & 0.9 & M & 0.84 & 0.27 & 105374 & 105374 & 9911 & 9016 & 0.720 & 0.720 & 0.003 & 0.000 \\
\hline 8000 & 1.0 & 1.0 & M & 0.93 & 0.27 & 72663 & 72663 & 6603 & 5773 & 0.705 & 0.705 & 0.003 & 0.000 \\
\hline 8000 & 1.0 & 1.1 & M & 1.03 & 0.26 & 50701 & 50701 & 4509 & 3695 & 0.682 & 0.683 & 0.008 & 0.001 \\
\hline 8000 & 1.0 & 1.2 & M & 1.12 & 0.26 & 36358 & 36358 & 3218 & 2388 & 0.649 & 0.651 & 0.007 & 0.001 \\
\hline 8000 & 1.0 & 1.3 & M & 1.21 & 0.29 & 26764 & 26764 & 2425 & 1596 & 0.612 & 0.612 & 0.024 & 0.001 \\
\hline 9500 & 0.4 & 0.8 & $\mathrm{~S}$ & 0.76 & 0.26 & 43810 & 695587 & 14847 & 12884 & 0.752 & 0.752 & 0.023 & 0.002 \\
\hline 9500 & 0.4 & 0.9 & $\mathrm{~S}$ & 0.85 & 0.27 & 27922 & 544208 & 9479 & 7438 & 0.751 & 0.709 & 0.037 & 0.002 \\
\hline 9500 & 0.4 & 1.0 & $\mathrm{~S}$ & 0.94 & 0.28 & 18612 & 418946 & 6367 & 4278 & 0.751 & 0.680 & 0.052 & 0.006 \\
\hline 9500 & 0.4 & 1.1 & $\mathrm{P}$ & 1.04 & 0.28 & 12867 & 313659 & 4403 & 2057 & 0.313 & 0.313 & 0.069 & 0.011 \\
\hline 9500 & 0.4 & 1.2 & $\mathrm{P}$ & 1.13 & 0.29 & 9181 & 221923 & 3151 & 235 & 0.047 & 0.069 & 0.079 & 0.017 \\
\hline 9500 & 0.6 & 0.8 & $\mathrm{~S}$ & 0.75 & 0.24 & 72663 & 364938 & 15051 & 13767 & 0.751 & 0.750 & 0.015 & 0.001 \\
\hline 9500 & 0.6 & 0.9 & $\mathrm{~S}$ & 0.85 & 0.25 & 46528 & 236659 & 1009 & 8353 & 0.751 & 0.729 & 0.500 & 0.025 \\
\hline 9500 & 0.6 & 1.0 & $\mathrm{~S}$ & 0.94 & 0.25 & 31103 & 178559 & 6412 & 5105 & 0.723 & 0.705 & 0.022 & 0.002 \\
\hline 9500 & 0.6 & 1.1 & $\mathrm{P}$ & 1.03 & 0.26 & 21571 & 138218 & 4469 & 3073 & 0.528 & 0.528 & 0.028 & 0.003 \\
\hline 9500 & 0.6 & 1.2 & $\mathrm{P}$ & 1.12 & 0.28 & 15409 & 105374 & 3247 & 1695 & 0.414 & 0.415 & 0.031 & 0.004 \\
\hline 9500 & 0.6 & 1.3 & $\mathrm{P}$ & 1.21 & 0.30 & 11298 & 79734 & 2420 & 621 & 0.235 & 0.235 & 0.032 & 0.007 \\
\hline 9500 & 0.8 & 0.8 & $\mathrm{~S}$ & 0.75 & 0.26 & 109778 & 202645 & 15447 & 14171 & 0.729 & 0.728 & 0.006 & 0.000 \\
\hline 9500 & 0.8 & 0.9 & $\mathrm{~S}$ & 0.84 & 0.25 & 72663 & 150804 & 9702 & 8612 & 0.722 & 0.720 & 0.005 & 0.000 \\
\hline 9500 & 0.8 & 1.1 & M & 1.02 & 0.28 & 33896 & 78905 & 4632 & 3545 & 0.620 & 0.631 & 0.010 & 0.002 \\
\hline 9500 & 0.8 & 1.2 & $\mathrm{P}$ & 1.12 & 0.28 & 24284 & 57012 & 3279 & 2173 & 0.557 & 0.557 & 0.014 & 0.002 \\
\hline 9500 & 0.8 & 1.3 & $\mathrm{P}$ & 1.20 & 0.30 & 17831 & 41997 & 2336 & 1285 & 0.470 & 0.470 & 0.050 & 0.003 \\
\hline 9500 & 1.0 & 0.8 & M & 0.75 & 0.27 & 150804 & 150804 & 15638 & 14353 & 0.724 & 0.724 & 0.002 & 0.000 \\
\hline 9500 & 1.0 & 0.9 & M & 0.84 & 0.27 & 105374 & 105374 & 9974 & 8906 & 0.713 & 0.713 & 0.003 & 0.000 \\
\hline 9500 & 1.0 & 1.0 & M & 0.93 & 0.28 & 72663 & 72663 & 6663 & 5653 & 0.695 & 0.695 & 0.004 & 0.000 \\
\hline 9500 & 1.0 & 1.1 & M & 1.03 & 0.27 & 50701 & 50701 & 4584 & 3588 & 0.667 & 0.667 & 0.007 & 0.001 \\
\hline 9500 & 1.0 & 1.2 & M & 1.12 & 0.27 & 36358 & 36358 & 3271 & 2264 & 0.627 & 0.631 & 0.010 & 0.001 \\
\hline 9500 & 1.0 & 1.3 & M & 1.21 & 0.26 & 26764 & 26764 & 2368 & 1410 & 0.574 & 0.579 & 0.032 & 0.001 \\
\hline 11000 & 0.4 & 0.8 & $\mathrm{~S}$ & 0.76 & 0.28 & 43810 & 695587 & 14999 & 12605 & 0.751 & 0.746 & 0.028 & 0.002 \\
\hline 11000 & 0.4 & 0.9 & $\mathrm{~S}$ & 0.85 & 0.25 & 27922 & 544208 & 9333 & 7069 & 0.751 & 0.710 & 0.054 & 0.005 \\
\hline 11000 & 0.4 & 1.0 & $\mathrm{P}$ & 0.94 & 0.28 & 18612 & 418946 & 6329 & 3858 & 0.420 & 0.420 & 0.059 & 0.006 \\
\hline 11000 & 0.4 & 1.1 & $\mathrm{P}$ & 1.03 & 0.30 & 12867 & 313659 & 4484 & 1377 & 0.211 & 0.211 & 0.036 & 0.011 \\
\hline 11000 & 0.6 & 0.8 & $\mathrm{~S}$ & 0.75 & 0.25 & 72663 & 364938 & 15139 & 13640 & 0.750 & 0.749 & 0.015 & 0.001 \\
\hline 11000 & 0.6 & 0.9 & $\mathrm{~S}$ & 0.85 & 0.26 & 46528 & 236659 & 9674 & 8250 & 0.750 & 0.737 & 0.023 & 0.002 \\
\hline 11000 & 0.6 & 1.0 & M & 0.94 & 0.27 & 31103 & 178559 & 6499 & 4849 & 0.696 & 0.536 & 0.010 & 0.002 \\
\hline 11000 & 0.6 & 1.1 & $\mathrm{P}$ & 1.03 & 0.25 & 21571 & 138218 & 4434 & 2770 & 0.480 & 0.480 & 0.030 & 0.003 \\
\hline 11000 & 0.6 & 1.2 & $\mathrm{P}$ & 1.12 & 0.29 & 15409 & 105374 & 3264 & 1322 & 0.336 & 0.336 & 0.026 & 0.005 \\
\hline 11000 & 0.6 & 1.3 & $\mathrm{P}$ & 1.21 & 0.29 & 11298 & 79734 & 2383 & 306 & 0.104 & 0.134 & 0.071 & 0.009 \\
\hline 11000 & 0.8 & 0.8 & $S$ & 0.75 & 0.26 & 109778 & 202645 & 15514 & 14031 & 0.724 & 0.724 & 0.005 & 0.000 \\
\hline 11000 & 0.8 & 0.9 & $\mathrm{~S}$ & 0.84 & 0.27 & 72663 & 150804 & 9928 & 8636 & 0.717 & 0.713 & 0.005 & 0.000 \\
\hline 11000 & 0.8 & 1.1 & $\mathrm{P}$ & 1.02 & 0.30 & 33896 & 78905 & 4696 & 3407 & 0.596 & 0.596 & 0.012 & 0.002 \\
\hline 11000 & 0.8 & 1.2 & $\mathrm{P}$ & 1.12 & 0.27 & 24284 & 57012 & 3257 & 1940 & 0.516 & 0.516 & 0.015 & 0.002 \\
\hline 11000 & 0.8 & 1.3 & $\mathrm{P}$ & 1.21 & 0.28 & 17831 & 41997 & 2401 & 1026 & 0.407 & 0.407 & 0.019 & 0.002 \\
\hline 11000 & 1.0 & 0.8 & M & 0.75 & 0.27 & 150804 & 150804 & 15694 & 14943 & 0.720 & 0.720 & 0.000 & 0.000 \\
\hline
\end{tabular}


E. Glebbeek and O. R. Pols: A grid of low-mass collisions, Online Material p 6

Table 5. continued.

\begin{tabular}{|c|c|c|c|c|c|c|c|c|c|c|c|c|c|}
\hline$t$ & $q$ & $M$ & Case & $M_{\text {remnant }}$ & $\frac{(1+q)^{2}}{q} \phi$ & $\tau_{\mathrm{ms}, 1}$ & $\tau_{\mathrm{ms}, 2}$ & $\tau_{\mathrm{ms}}$ & $t_{\mathrm{ms}}$ & $X_{\mathrm{c}, 0}$ & $X_{\mathrm{c}, \mathrm{zms}}$ & $\Delta \log _{10} L$ & $\Delta \log _{10} T_{\text {eff }}$ \\
\hline 11000 & 1.0 & 0.9 & $\mathrm{M}$ & 0.84 & 0.28 & 105374 & 105374 & 10036 & 751 & 0.706 & 0.706 & 0.000 & 0.000 \\
\hline 11000 & 1.0 & 1.0 & M & 0.93 & 0.28 & 72663 & 72663 & 6723 & 5559 & 0.685 & 0.685 & 0.003 & 0.000 \\
\hline 11000 & 1.0 & 1.1 & M & 1.02 & 0.28 & 50701 & 50701 & 4639 & 3473 & 0.653 & 0.653 & 0.007 & 0.001 \\
\hline 11000 & 1.0 & 1.2 & M & 1.11 & 0.29 & 36358 & 36358 & 3332 & 2125 & 0.606 & 0.606 & 0.011 & 0.001 \\
\hline 11000 & 1.0 & 1.3 & M & 1.21 & 0.29 & 26764 & 26764 & 2321 & 1175 & 0.541 & 0.545 & 0.025 & 0.001 \\
\hline 12500 & 0.4 & 0.9 & $\mathrm{~S}$ & 0.85 & 0.27 & 27922 & 544208 & 9492 & 6505 & 0.750 & 0.690 & 0.014 & 0.004 \\
\hline 12500 & 0.4 & 1.0 & $\mathrm{P}$ & 0.94 & 0.27 & 18612 & 418946 & 6304 & 3365 & 0.355 & 0.355 & 0.067 & 0.007 \\
\hline 12500 & 0.4 & 1.1 & $\mathrm{P}$ & 1.03 & 0.29 & 12867 & 313659 & 4440 & 492 & 0.095 & 0.096 & 0.047 & 0.016 \\
\hline 12500 & 0.6 & 0.8 & $\mathrm{~S}$ & 0.75 & 0.26 & 72663 & 364938 & 15230 & 13508 & 0.750 & 0.747 & 0.017 & 0.001 \\
\hline 12500 & 0.6 & 0.9 & $\mathrm{~S}$ & 0.85 & 0.24 & 46528 & 236659 & 9475 & 7857 & 0.748 & 0.718 & 0.025 & 0.002 \\
\hline 12500 & 0.6 & 1.0 & M & 0.94 & 0.25 & 31103 & 178559 & 6399 & 4582 & 0.546 & 0.546 & 0.027 & 0.002 \\
\hline 12500 & 0.6 & 1.1 & $\mathrm{P}$ & 1.03 & 0.28 & 21571 & 138218 & 4537 & 2526 & 0.433 & 0.433 & 0.029 & 0.004 \\
\hline 12500 & 0.6 & 1.2 & $\mathrm{P}$ & 1.12 & 0.30 & 15409 & 105374 & 3301 & 918 & 0.249 & 0.249 & 0.022 & 0.007 \\
\hline 12500 & 0.8 & 0.8 & S & 0.75 & 0.27 & 109778 & 202645 & 15605 & 13908 & 0.720 & 0.719 & 0.007 & 0.000 \\
\hline 12500 & 0.8 & 0.9 & $\mathrm{~S}$ & 0.84 & 0.28 & 72663 & 150804 & 10004 & 8516 & 0.712 & 0.706 & 0.007 & 0.000 \\
\hline 12500 & 0.8 & 1.0 & M & 0.94 & 0.26 & 48790 & 109778 & 6515 & 5108 & 0.630 & 0.633 & 0.008 & 0.001 \\
\hline 12500 & 0.8 & 1.1 & $\mathrm{P}$ & 1.03 & 0.27 & 33896 & 78905 & 4552 & 3115 & 0.567 & 0.567 & 0.013 & 0.002 \\
\hline 12500 & 0.8 & 1.2 & $\mathrm{P}$ & 1.11 & 0.29 & 24284 & 57012 & 3326 & 1796 & 0.476 & 0.476 & 0.016 & 0.002 \\
\hline 12500 & 0.8 & 1.3 & $\mathrm{P}$ & 1.21 & 0.27 & 17831 & 41997 & 2366 & 817 & 0.338 & 0.338 & 0.027 & 0.002 \\
\hline 12500 & 1.0 & 0.8 & M & 0.75 & 0.23 & 150804 & 150804 & 15091 & 13462 & 0.712 & 0.711 & 0.003 & 0.000 \\
\hline 12500 & 1.0 & 0.9 & M & 0.84 & 0.28 & 105374 & 105374 & 10100 & 8680 & 0.699 & 0.699 & 0.004 & 0.000 \\
\hline 12500 & 1.0 & 1.0 & M & 0.94 & 0.25 & 72663 & 72663 & 6464 & 5187 & 0.672 & 0.672 & 0.003 & 0.000 \\
\hline 12500 & 1.0 & 1.1 & M & 1.02 & 0.29 & 50701 & 50701 & 4705 & 3866 & 0.639 & 0.639 & 0.003 & 0.001 \\
\hline 12500 & 1.0 & 1.2 & M & 1.12 & 0.26 & 36358 & 36358 & 3235 & 1911 & 0.576 & 0.580 & 0.012 & 0.001 \\
\hline 12500 & 1.0 & 1.3 & M & 1.21 & 0.27 & 26764 & 26764 & 2376 & 1126 & 0.502 & 0.507 & 0.049 & 0.002 \\
\hline
\end{tabular}

